\title{
Acquisition of uropygial gland microbiome by hoopoe nestlings
}

Manuel Martín-Vivaldia ${ }^{\mathrm{a} \text {, }}$, Juan José Soler ${ }^{\mathrm{b}}$, Ángela Martínez-García ${ }^{\mathrm{b}}$, Laura Arco ${ }^{\mathrm{a}}$, Natalia Juárez-García-Pelayo a , Magdalena Ruiz-Rodríguez ${ }^{\mathrm{b}}$, Manuel Martínez-Bueno ${ }^{\mathrm{c}}$

ORCID: orcid.org/0000-0002-5432-425X; orcid.org/0000-0003-2990-1489; orcid.org/0000-0002$4202-5180$

a Departamento de Zoología, Universidad de Granada, E-18071 Granada, Spain;

${ }^{\mathrm{b}}$ Estación Experimental de Zonas Áridas (CSIC) E-04120 Almería, Spain;

c Departamento de Microbiología, Universidad de Granada, E-18071 Granada, Spain;

* Correspondence author. e-mail: $\underline{\operatorname{mmv} @ \text { ugr.es }}$

Keywords: byproduct cooperation, coevolution, mutualism, preen gland, symbiotic bacteria Word count: 6724 without references

Running headline: Uropygial microbiome acquisition

Acknowledgements. Jonathan Romero, Manuel Soto, Jorge Doña, Ana Belén García, Alicia Astasio, Elisa García, Rosa María Morales and Marcos Gómez helped in caring for captive hoopoes. Sonia Rodríguez-Ruano performed the DNA extraction of the nestling secretions. Juan Manuel Peralta and two anonymous referees revised a previous version of the MS. Support by funding was provided by Spanish Ministerio de Ciencia e Innovación, European funds (FEDER) (CGL2009-14006, CGL2010- 19233-C03-01, CGL2010-19233-C03-03, CGL2013-48193-C3-1-P, CGL2013-48193-C3-2-P) and Junta de Andalucía (P09- RNM-4557). AM-G had a predoctoral grant from the Junta de Andalucía (P09-RNM-4557). 


\section{Abstract}

Mutualistic symbioses between animals and bacteria depend on acquisition of appropriate symbionts while avoiding exploitation by non-beneficial microbes. The mode of acquisition of symbionts would determine, not only the probability of encountering, but also evolutionary outcomes of mutualistic counterparts. The microbiome inhabiting the uropygial gland of the European hoopoe (Upupa epops) includes a variety of bacterial strains, some of them providing antimicrobial benefits. Here, the mode of acquisition and stability of this microbiome is analyzed by means of Automated rRNA Intergenic Spacer Analysis and two different experiments. The first experiment impeded mothers' access to their glands, thus avoiding direct transmission of microorganisms from female to offspring secretions. The second experiment explored the stability of the microbiomes by inoculating glands with secretions from alien nests. The first experiment provoked a reduction in similarity of microbiomes of mother and nestlings. Interestingly, some bacterial strains were more often detected when females had not access to their glands, suggesting antagonistic effects among bacteria from different sources. The second experiment caused an increase in richness of the microbiome of receivers in terms of prevalence of Operational Taxonomic Units (OTUs) that reduced differences in microbiomes of donors and receivers. That occurred because OTUs that were present in donors but not in receivers incorporated to the microbiome of the latter, which provoked that cross-inoculated nestlings got similar final microbiomes that included the most prevalent OTUs. The results are therefore consistent with a central role of vertical transmission in bacterial acquisition by nestling hoopoes, and support the idea that the typical composition of the hoopoe gland microbiome is reached by the incorporation of some bacteria during the nestling period. This scenario suggests the existence of a coevolved core microbiome composed by a mix of specialized vertically transmitted strains and facultative symbionts able to coexist with them. The implications of this mixed mode of transmission for the evolution of the mutualism are discussed. 


\section{Introduction}

The life of every animal is conditioned by interactions with an extremely high variety of ubiquitous bacteria that are able of colonizing and exploiting any nutritional resource in any chemical form [1]. Many of those potential partners are enemies, while some others do not affect the host, or even provide benefits. Evolutionary processes should therefore select for strategies that reduce encounters with parasitic microorganisms; and increase the probability of recruitment of the beneficial ones [2]. Benefits of the association with bacteria can have multiple forms: from nutritive resources to defensive products []ㅡ or inactivation of toxic residues [4]. Hosts provide beneficial microorganisms with physical support and resources for growth, which could also be exploited by undesired parasitic-symbiont partners (e.g. [5] $)$. Even though the risk of exploitation of host resources by non-mutualistic symbionts is considered a destabilizing force of mutualistic relationships (reviewed in [6] ), mutualisms with bacteria are ubiquitous and remain during evolutionary time [4] indicating the existence of mechanisms preventing parasitic exploitation.

One of the main questions in evolutionary ecology is to disentangle mechanisms explaining mutualistic coevolutionary relationships while preventing the intromission of the surrounding potential exploiters. Several studies have modelled the stability of mutualisms and reviewed the current evidence for these systems to be stable [7-11]. In this sense, vertical transmission from parents would reduce the risk of exploitation and provide descendants with an adequate pool of collaborators $[\underline{2}, 12-15]$. However, hosts that acquire their mutualistic symbionts horizontally are more compromised by parasites' exploitation because they need to recruit appropriated symbionts from the surroundings, which depend upon availability $[\underline{16}, 17]$. Indeed, they need to collect beneficial strains while avoiding colonization by parasites ("partner choice" [18]). Such selection procedures involve recognition, attachment, regulation of immune responses, and control of nutrient release for the symbiont $[\underline{17}, 19, \underline{20}]$. Horizontal transmission may also imply some advantages since it allows a plastic selection of cooperators and the best option may differ for different environmental conditions (e.g. [21]). For instance, bacterial symbionts producing more antimicrobials that benefit hosts can be established automatically within the symbiont community 
of hosts ("byproduct cooperation" [ㄴ,22]]) and impede colonization of parasitic symbionts.

Therefore, even complex communities resulting from horizontal transmission reach stability, as shown by the microbiome of rumen of mammals that has demonstrated high inertia and resilience after perturbations, including the inoculation of alien communities [23]. Some models have even suggested the possibility that mixed communities, including both vertically and horizontally acquired strains, would further guarantee the stability of the association [24]. The scenarios described above, therefore, indicate that knowing the mode of acquisition of symbionts is essential for understanding functioning, stability and evolution of mutualistic associations.

An interesting model for exploring the importance of mechanisms of symbiont acquisition is the system formed by the hole-nester European hoopoe (Upupa epops) and the bacteria living in its uropygial gland [25-27]. The uropygial secretion of Upupiformes (hoopoes and their relative woodhoopoes) is special (dark and odorous) due to the presence of symbiotic bacteria in the gland $[\underline{28}, \underline{29}]$. In the case of the hoopoes, their symbionts change the composition of the oil produced by the bird, generate metabolites with antimicrobial properties [30-33], and positively affect hatching success [27] when the female voluntarily impregnates their eggshell during incubation [ $\underline{34}, \underline{35}]$. The special secretions with bacteria are only maintained during the stay within the nest-hole of females and nestlings [29] and, therefore, must be acquired every breeding season. Results of previous Automated rRNA Intergenic Spacer Analysis (ARISA) showed that both females and nestlings host a bacterial community formed by a group of eight highly prevalent strains accompanied by a long list of Operational Taxonomic Units (OTUs) in a range of frequencies of appearance from $50 \%$ to $10 \%$ [36]. Moreover, cross-fostering experiments suggested that nestlings are able to acquire new symbiont strains when moved to a different nest $[\underline{36}, \underline{37}]$. In this way, the stability of the bacterial community could depend on the possibility of encountering new strains and, thus, vertical and horizontal acquisition of symbionts may explain microbiomes of nestlings $[\underline{36}, \underline{37}]$. However, the factors affecting the dynamic of colonization of the gland by bacterial symbionts are not known, and differences in competitive ability of different bacterial strains reaching the uropygial gland are supposed to affect their success colonizing and growing in the uropygial secretion [38]. This 

coming from different nest environments $[\underline{16}, \underline{39}]$. Such experiment will also allow identifying particular bacterial strains able to colonize uropygial glands where they were not present.

In this context, glands of nestlings close to fledge were inoculated with secretion from alien nestlings that acquired their microbiome in different nests. Assuming some level of vertical transmission of symbionts, to increase differences among donor and receptor of inocula, crossinoculations were performed between nestlings from nests with mothers that had full or experimentally restricted access to their uropygial gland. Impeding bird access to their glands by covering them has previously been used with success to test effects of uropygial secretions in several studies $[\underline{34}, \underline{35}, \underline{40}, \underline{41}]$. We have used here such an approach as a reversible alternative to the extirpation of glands (e.g. [42]). In this way, two crossed experimental treatments were performed: (1) restriction of gland access for females and (2) cross-inoculation of nestling secretions. Thus, only a fraction of experimentally inoculated nestlings had the possibility of acquiring bacterial strains from their mothers' glands. This experimental approach also allow testing the influence of vertical transmission (i.e. female treatment) on the effect of experimental inoculation of nestling uropygial glands.

With this experimental design, the following hypotheses and predictions were tested: (a) there is vertical transmission of bacteria directly from female to offspring glands if the composition of microbiomes of nestlings is affected by experimental covering of female glands. (b) If hoopoes harbor a particular co-evolved microbial assemblage in secretions, the cross-inoculation experiment should result in the colonization of glands by complementary bacterial strains, commonly detected in hoopoes. This should particularly be the case in nests with experimental females where the vertical transmission of commonly detected strains to uropigial gland of nestlings is restricted. This scenario also predicts an increase in microbiome similarity of secretions after the inoculation experiment. (c) On the other hand, if different communities are the result of different optimal microbiomes for different individuals (see [23]), there should be evidence of stability (i.e., no effects of cross-inoculation on similarity) and a limited probability of colonization by new bacterial 
strains. (d) Finally if different strains differ in the ability to colonize and establishing in the microbiome of the uropygial gland, we should find that the effects of experimental restriction of vertical transmission or the inoculation experiment on particular microorganisms, will depend on these abilities (i.e. identity). 
Two experimental approaches were used; one to restrict the vertical transmission of bacterial communities from females to offspring, and the other to detect possible changes in the microbiome composition of the uropygial secretion of nestlings along their stay in the nest after inoculation with alien secretions.

In Experiment 1, females' access to the uropygial gland was manipulated from the start of laying until they finished brooding (Fig 1a, see [34] for details of the method for covering female glands). Thus, experimental females could not use the uropygial secretion to cover eggs $[\underline{34}, \underline{35]}$ or to preen nestlings or themselves [36], and the direct or indirect transmission of bacteria from female gland to those of their nestlings was impeded. Two groups of control birds were established. Control I females were provided with a similar structure as experimental females, but not covering the gland entrance and thus not preventing normal access to secretions. A third group of breeding females (control II) were visited and handled at the same rate as those of experimental and control I groups. Females were assigned to a particular treatment in sequence by laying date, alternating experimental with one of the two control treatments, which were alternatively selected to get a similar sample size between experimental females and both control groups combined. Treatments were balanced within sub-populations. In order to test the effect of the experiment on the transmission of symbiotic bacteria from mother to offspring, the microbiomes of their uropygial glands were compared. Female glands were sampled on the day of start of laying, before manipulation, and those of nestlings on the $16^{\text {th }}$ day of the nestling period (Fig. 1a).

Experiment 2 was performed with 16 days old chicks of mothers that were subject to the Experiment 1. After sampling secretions, half of the nestlings in each brood (Controls) were inoculated with $3 \mu 1$ of their own secretion with a sterile micropipette within the papilla of the gland, while the other half of the brood (Experimentals) were inoculated with $3 \mu$ of secretion proceeding from nestlings of a different nest. Except for the few cases in which wild broods were 
used as donors of secretions, mothers of donor nestlings were under different experimental treatment than those of receivers. The $3 \mu$ of secretion used for inoculation were pipetted from a sterile microfuge tube where the available secretion of each nestling was previously homogenized. Nests were paired by hatching date so that secretions were interchanged between nestlings of 

Agar (TSA) medium. Plates were inoculated, within the same day of sampling, with $5 \mu$ of serial dilutions of the secretions in sterile Phosphate Buffer Saline (PBS), and cultures were incubated aerobically at $37{ }^{\circ} \mathrm{C}$ for $24 \mathrm{~h}$. Bacterial load was expressed as number of colony-forming units

\section{Molecular analyses}

Bacterial DNA was extracted from uropygial secretions with a commercial KIT (The FavorPrep ${ }^{\mathrm{TM}}$ (CFUs) per $\mu 1$ of secretion. Blood Genomic DNA Extraction Kit, Favorgen). Automated rRNA Intergenic Spacer Analysis (ARISA) was used to characterize the composition of bacterial communities (see Supplemental Material-I for details). The purpose here is not to describe the composition of hoopoe uropygial microbiomes, but explore influence of mothers (i.e. vertical transmission) and of later colonization (i.e. inoculation) on the microbiome of nestling hoopoes, with particular emphasis on bacteria of known beneficial effects (i.e. enterococci). Thus, the use of ARISA is appropriate here. Furthermore, particular OTUs from ARISA have previously been suggested to interact with the life history characteristics of hoopoes $[\underline{36}, 46,47]$, which will allow interpretation of results of particular OTUs in the performed experiment in relation to what is already known of their role in these communities. Given that it is possible that different fragment lengths came from identical bacterial strains or species, except when indicated we use them as Operational Taxonomic Units (OTUs) of unknown taxonomic affiliation. The generated presence-absence matrix was used in all analyses.

The hoopoe nestlings involved in the inoculation experiment harbored a total of 82 OTUs, 49 of which were present only in up to three individuals before the experimental inoculation of their glands. For the calculation of richness only the 33 OTUs present in at least four of those samples were considered [47]. Moreover, to understand the dynamic of the main assemblages naturally encountered in hoopoes, and for analyses considering differences in composition among bacterial communities, only those with at least $50 \%$ prevalence were considered (for this calculation we only included samples of females and the first available for nestlings of control females). There were 16 
OTUs fulfilling this criterion, which are named by the length of their ITS fragment (OTUs 182, $242,254,278,306,310,326,330,346,350,406,422,466,474,534$ and 566), which hereafter are referred as the "core microbiome" of secretions. The main effects of both experiments on microbiome composition did not change if we use the 33 OTUs with prevalence $>3$ instead.

\section{Statistical analyses}

Three different estimates have been used characterizing the microbiome of the uropygial secretion

To detect possible opposed effects of experiments on different components of the microbial communities, sample positions in the multiscale Jaccard distance space were decomposed in two or three first axes of Principal Coordinates Analyses (PCo). The coordinates (i.e. scores) of each sample were used as dependent variables and the statistical tests performed with Primer7.

Before the inoculation treatments, bacterial load, richness and composition of microbiomes of the secretion of nestlings in nests of females under the two control treatments did not differ significantly (GLMs, after controlling for nest identity nested within experimental treatment as random factor, bacterial load: $\mathrm{F}(1,9)=0.001, \mathrm{p}=0.97$; richness: $\mathrm{F}(1,9)=1.44, \mathrm{p}=0.269$; microbiome composition PCo1: $\mathrm{F}(1,9)=0.019, \mathrm{p}=0.894$; $\mathrm{PCo} 2 \mathrm{~F}(1,9)=0.958, \mathrm{p}=0.360)$. Thus, data of the broods of control-I and control-II females were pooled in a single group of control nests in subsequent analyses.

The effect of female treatment on similarity between nestling and female microbiomes, as well as the effect of the inoculation experiment on microbiomes of initial and final nestling samples were explored in repeated measures ANOVAs. The fixe effect of treatment was included as 
for Experiment 2), and type of individual (i.e., nestling or female) and time of sampling, respectively, as the within fixed factors. For analyses related to Experiment 1, the repeated factor tests for differences between females and nestlings in PCo scores. For those related to Experiment 2, the repeated factor compared PCo scores between the initial and final samples (those obtained one week after inoculation) of nestlings.

To estimate the dispersion of samples within the groups of cross-inoculated and control nestlings in the PCo space, differences in the deviations from the median of each group were

Bacterial loads of secretion of 16 days old nestlings did not differ between years (GLM after controlling for nest identity nested within year $(\operatorname{GLM}, F(1,17)=2.58, p=0.126)$, nor did PCo1 scores $(\mathrm{GLM}, \mathrm{F}(1,17)=1.32, \mathrm{p}=0.268)$, although the effect of year on PCo2 scores approached significance $(\mathrm{GLM}, \mathrm{F}(1,17)=3.63, \mathrm{p}=0.076)$. Since results for PCo scores are qualitatively identical independent of inclusion of year as an additional independent factor, only results of models that do not include year identity are shown. However, richness of bacterial community of nestlings at this age were lower in 2012 than in $2010(\mathrm{GLM}, \mathrm{F}(1,17)=6.18, \mathrm{p}=0.024)$ and, thus, year identity was included in the analyses explaining variation in richness.

Neither richness, nor bacterial load (CFUs/ $\mu$ l), or PCo scores differed among subpopulations (GLMs, all $\mathrm{p}>0.05$ ) and treatments were balanced within them. Thus, subpopulation identity was not included in the analyses. Moreover, nestlings sharing the same nest have identical values for female related variables (e.g. bacterial load in CFUs/ $\mu$ l) and, to account for non-independence of 
information of nestlings within the same nest, the degrees of freedom were adjusted to number of sampled nests.

Finally, for analyses exploring similar association for different OTUs, the false-discovery-rate

\section{Results}

\section{$\underline{\text { Bacterial richness }}$}


The composition of nestlings' core microbiomes differed from those of their mothers (repeated measures MANOVA with PCo1 and PCo2 scores: R1, Wilks $=0.194, \mathrm{~F}(2,15)=64.4, \mathrm{p}$ $<0.0001)$. In addition, microbiomes were more similar between control females and their nestlings treatment, Wilks $=0.781, \mathrm{~F}(2,15)=4.33, \mathrm{p}=0.033)$. Mostly, these effects were due to differences related to PCo2 (Table 2, Fig. 4a). Scores of this PCo explained $19.1 \%$ of total variance of the microbiome composition of samples (Fig. 4b).

The inoculation experiment also affected the composition of the microbiome present in nestling glands (repeated measures MANOVA with PCo1, PCo2 and PCo3 scores: R1 (paired comparison before-after inoculation), Wilks $=0.645, \mathrm{~F}(3,32)=5.88, \mathrm{p}=0.003)$. The microbiomes of experimental nestlings (cross-inoculated with secretions from a different nest) experienced larger changes than those of control nestlings (repeated measures MANOVA: R1* nestling treatment, Wilks $=0.781, \mathrm{~F}(3,32)=4.44, \mathrm{p}=0.010)$. The effects of the inoculation experiment were due to differences in PCo1 and PCo2 scores (Table 3, Fig. 5). Moreover, final among-individuals variation was higher for control (deviations from the median of each group mean(SE) $=46.7(3.8)$ ) than for experimental $(\operatorname{mean}(\mathrm{SE})=20.2(5.0))$ nestlings $($ PermDisp, $\mathrm{F}(1,34)=17.70, \mathrm{p}<0.001)$. The microbiome composition of the final samples of cross-inoculated nestlings showed a higher level of similarity among them (Simper, average similarity $=77.46 \%$, with 10 OTUs of the core microbiome with a probability of presence $>80 \%$ ) than among those of control nestlings (average similarity $44.84 \%$, only 4 OTUs with a probability of occurrence $>60 \%$ ). Indeed, samples of cross-inoculated nestlings appeared close to each other in the PCo space (Fig. 5), in the area that correlates with a higher number of OTUs (Fig. 4 in Supplemental material-II).

For the subsample of nests with information on the microbiome of donors and receivers, differences in their PCol scores positively correlated with the changes in microbiome composition caused by the inoculation experiment (only experimental nestlings, Linear Regression $\mathrm{F}(1,13)=$ 

scores $\left(\mathrm{F}(1,13)=4.56, \mathrm{p}=0.052, \mathrm{R}^{2}=0.260\right)$.

\section{$\underline{\text { Cultivable bacterial density }}$}

Bacterial density of nestling secretions at 16 days of age was positively correlated with that of

\section{Experimental effects on particular OTUs}

Six OTUs of nestling secretions were significantly affected by the experiments. The covering of female glands affected the presence of four OTUs in the secretion of nestlings. In nestlings of mothers that had not access to their uropygial gland, prevalence of OTUs 346 and 466 decreased, while that of OTUs 306 and 406 increased (Table 5). Moreover, the presence of the OTU566 in nestlings was not affected by female manipulation, but was positively associated with its detection in their mothers, either experimental or control (Table 5). For all these OTUs, but also for most in the core microbiome, the effect of the inoculation experiment depended on microbiome composition of donors and receivers. Whenever an OTU was present in the donor and not in the receiver, the latter incorporated to the nestling secretion. In addition, there was an association between the cultivable bacterial density in secretions and the presence of particular OTUs in their 
microbiome. It was higher for secretions harboring OTU306, while presence of OTUs 242, 346 and 566 was negatively related to cultivable bacterial density (summary of results in Table 5, analyses in Supplemental Material-II).

\section{Discussion}

Experimental results support for the first time the hypothesis that the microbiome hosted in the uropygial gland of nestling hoopoes is in part transmitted vertically from mother to offspring, but also that nestling microbiomes maintain their ability to incorporate new strains during the entire nesting period. These results have important consequences for the understanding of the dynamic and evolution of the relationships between hoopoes and their uropygial gland symbionts.

The detected effects of covering female glands support the influence of female uropygial secretion conforming the microbiome of that of their offspring. Differences between nestlings and mothers in microbiome composition and cultivable bacterial density were significantly larger for nests of females with impeded access to their glands. Previous studies performing cross-fostering experiments already showed the existence of genetic effects (i.e. nest of origin) on the composition of the microbiome of hoopoe nestlings $[\underline{36}, \underline{37}]$. However, similarities due to nest of origin might be due to direct transmission from females to offspring or common acquisition by mothers and nestlings, and cross-fostering experiments of nestlings do not allow to distinguish between these two possibilities. Results presented here definitely show that vertical transmission is in fact responsible of the previously detected effect of nest of origin, at least partially. This is an important result, because vertical transmission of those strains assures that part of the microbial community that nestlings harbor in their glands come from an individual (their mother), which has already been successful in surviving and breeding, thus transferring an optimized microbiota adapted to their particular environment $[2,13,14]$. It is well established that the evolution of vertically transmitted symbionts selects for characteristics that benefit both hosts and symbionts [15]. Moreover, when hosts benefits are mediated by antibiotic production, as it occurs in hoopoes [27,31-34], the vertical 
transmission of one antibiotic producing strain would constraint the recruitment of non-resistant ones and favor recruitment of other antibiotic producers [24]. The existence of several bacterial strains co-transmitted vertically from mother to offspring could also imply the evolution of tolerance, co-operation or resource dependence among them [49]. Vertical transmission also affects the evolution of the symbiotic relationship among counterparts [2,15]. Whenever symbionts complete their life cycle within a host species, and their fitness become close related to that of their host, the coevolutionary process provokes symbiont specialization on hosts as well as genetic barriers preventing gene flow among close relatives living in different hosts. Thus, similarly to what has been described in other systems [2], such process would lead to separated evolution among isolated populations of hosts, therefore predicting the existence of different microbial symbiont strains in different hoopoe populations, subspecies or species. However, particularities of the hoopoe-bacteria system may affect specialization and speciation processes of hoopoe symbionts. For instance, secretion of females drastically change during the non-breeding season (see below), which may imply either adaptations of the symbionts to resist that period, specialized body reservoirs in females, or ability to change of microhabitat within the female body. Future studies should explore such possibilities.

Interestingly, there were clear inter-nest differences in the microbiomes harbored by nestlings. This could be caused by a selective adaptive acquisition of bacteria [23], but also might be the consequence of differences in availability in nest environments. In accordance with the latter possibility, the experimental inoculation of secretions from a different nest provoked marked changes in richness and composition of microbiomes of nestlings' secretions after a particular community had been established along two thirds of their nestling cycle. Detected changes in the microbiome of nestlings due to inoculation were mainly explained by acquisition of strains present in the donor individual that were absent in the receiver secretion. Thus, after the experimental inoculations, communities of experimental nestlings from different nests became more similar to each other than those of control nestlings. These results suggest that there is a group of OTUs that are commonly found in hoopoe nestlings secretions and that conform to the typical microbiome. A 
similar effect was found for enterococci strains differing in bacteriocin production that varied in their presence among hoopoe nests [38].

The experimental approaches and results allow discussing the origin of particular OTUs of nestling secretions. When females could not use their secretions, many nestlings failed in harboring OTU346 and OTU466, suggesting female secretion is the main source of these two OTUs. Moreover, although detection of OTU566 in offspring was positively associated with its presence in females, the manipulation of female access to glands did not affect its acquisition by nestlings. Therefore, either, environmental conditions shared by nestlings and mother are responsible for the recruitment of this OTU in their uropygial secretions, or there is transmission from female to offspring by other via different of the female gland. We know from previous work [47] that prevalence of OTU566 in glands and cloacae of females as well as on the eggshells are very similar (55\%, 45\%, 35\% respectively). Something similar occurs with OTU306, which is especially common in female cloaca and on the eggshells ( $70 \%$ and $57 \%$ respectively, [47]) in comparison with prevalence in female uropygial gland (25\%,35\% in the present study). OTU306 (together with OTU406) characterize the microbiomes of offspring of females without access to their glands. All this evidence suggests that the cloaca of mothers is the most probable source of these bacteria (at least of the OTU566 and OTU306) for nestlings. We know that Enterococcus faecalis, by far the most prevalent cultivable bacteria species growing from hoopoe secretions $[\underline{27}, \underline{32}, \underline{38}]$, produces an ARISA peak at 306 (Antonio Martín-Platero, pers. comm.). Interestingly, OTU306 was the only OTU that was positively related to density of cultivable bacteria in the secretion of nestling hoopoes. Previous studies have also shown that E. faecalis, and some others Enterococcus strains isolated from hoopoe secretions, produce bacteriocins with an ample antimicrobial spectrum $[\underline{30}, \underline{32}, \underline{38}]$ and that their abundance in the secretion of female hoopoes is positively related to hatching success [34]. Thus, detecting the possible source of this OTU for hoopoe secretions is of particular interest and, casting light on this subject, the experimental results suggest that hoopoes recruit it from the cloacae to uropygial glands. 

on the presence of other bacteria. First, the experimental covering of female glands resulted in nestling microbiomes with increased prevalence of OTU306 and OTU406 and this suggests that particular OTUs transmitted vertically prevent their establishment in the uropygium of nestlings.

Second, this experiment also affected the mother-offspring relationship in the density of

cultivable bacteria. The abundance of cultivable bacteria in mother and offspring was related only for control females, while when the experiment impeded vertical transmission (experimental females), nestlings harbored greater loads of cultivable bacteria, and those loads were not predicted by that in mother glands. This suggests that the bacteria growing from nestlings' secretions of control females are a subset of those growing from nestlings'secretions in which vertical transmission has been impeded. Probably they are those with the potential to live in hoopoe secretions in presence of OTUs normally transmitted from female glands, while nestlings from experimental females harbor also less competitive strains able to grow in this experimental scenario of reduced competence. Given that OTU306 is the main predictor of cultivable bacteria load in secretions, this result can be interpreted in terms of abundance of OTU306. Since OTU306 probably represents mainly Enterococcus faecalis (see above), this possibility implies the existence of mechanisms selecting for particular Enterococci strains (i.e. those with higher antimicrobial potential).

Only three bird species are known to host symbiotic bacteria inside the uropygial gland in healthy individuals: the red-billed woodhoopoe (Phoeniculus purpureus), the European hoopoe and the turkey (Meleagris gallopavo, [50]). While in the turkey the influence of the symbionts (Corynebacterium uropygiale) has not yet been studied, in both Upupiform species, symbionts are responsible of several properties of the secretions including their defensive function $[29, \underline{51}, \underline{52}]$. In the red-billed woodhoopoe, all individuals maintain the symbiosis in the uropygial gland throughout the year, what has driven the evolution of a specific Enterococcus symbiont (E. phoeniculicula, [28]), although the whole community has never been studied by molecular methods in this system. In the case of European hoopoes, the association with bacteria in the gland is not 
continuous, but cyclic $[\underline{27}, \underline{29}]$. This probably has prevented to some extent the specialization of the symbiotic relationship $[\underline{5} 3, \underline{5} 4]$. Thus, the enterococci found in hoopoes are common in several environments [32] and from the results in the present study they probably are recruited from the cloaca. The microbiome of the uropygial secretion of hoopoes is more complex than that usually established in animal glands specialized in hosting symbionts (e.g. single actinobacteria species protecting fungus growing ants' gardens $[\underline{5} 5, \underline{5} 6])$. It includes a combination of 8 to 27 more frequent OTUs accompanied by up to 124 scarce OTUs with reduced prevalence [44], and results here suggest different sources for them. A few are inherited from mother to nestling gland, others may come either from female or nestling cloaca, and many others can apparently be obtained from environmental sources accessible within the nest-hole $[\underline{36}, \underline{37}, \underline{45}]$.

Despite such apparently complex microbiome, a more or less stable combination of OTUs is typical (core microbiome), and results suggest that the competitive ability of strains vertically transmitted from mothers gland differentially promote or restrict the establishment of others that are able of colonizing the nestling gland from other sources (see [24]). Previously, it was shown that the prevalence of different enterococci strains in the glands was related to their ability to produce antimicrobial bacteriocins [38]. All these results suggest that a mutualism based in byproduct cooperation $[\underline{4}, \underline{22}]$ determine the microbial composition of hoopoe uropygial gland microbiome.

Summarizing, hoopoe nestling uropygial glands harbor core microbiomes that to some degree vary in their composition, but, when experimentally put in contact, converge to the same combination of OTUs. The experiments further demonstrate the importance of vertical transmission determining the core microbiome of nestling glands, and suggest the existence of cloaca or gut reservoirs for particular OTUs. Further advance in the understanding of evolution of this system should address the determination of the taxonomic affiliation of these most common OTUs, in order to study their particular function in the symbiotic community, and finding the inter-annual reservoir for the vertically transmitted symbionts. 


\section{Ethical approval}

We performed the study following relevant Spanish national (Decreto 105/2011, April 19) and regional guidelines. The ethics committee of the Spanish National Research Council (CSIC) approved the protocol, and the Consejería de Medio Ambiente de la Junta de Andalucía, Spain, provided all necessary permits for nest and nestling manipulations (Ref: SGYB/FOA/AFR/CFS) as good as the establishment and maintenance of the captive breeding population (Resolution of April 14 2008).

\section{Conflict of Interest}

The authors declare that they have no conflict of interest.

\section{Data accessibility}

Data used in this paper can be found in the CSIC Institutional Repository. (Accession numbers still not available)

\section{Authors' contributions}

MM-V, JJS, AMG, MRR and MMB conceived the ideas and designed methodology; AMG, LA and NJG-P collected the data with considerable help from MM-V and JJS; MM-V and JJS analyzed the data; MM-V led the writing of the manuscript with considerable help by JJS. All authors contributed critically to the drafts and gave final approval for publication.

\section{References}

1. Moran NA (2002) The ubiquitous and varied role of infection in the lives of animals and plants. American Naturalist 160:S1-S8

2. Bright M, Bulgheresi S (2010) A complex journey: transmission of microbial symbionts. Nature Reviews Microbiology 8 (3):218-230 
3. Florez LV, Biedermann PHW, Engl T, Kaltenpoth M (2015) Defensive symbioses of animals with prokaryotic and eukaryotic microorganisms. Natural Product Reports 32 (7):904-936

4. Sachs JL, Skophammer RG, Regus JU (2011) Evolutionary transitions in bacterial symbiosis. Proceedings of the National Academy of Sciences of the United States of America 108:1080010807

5. Morales J, Kokkori S, Weidauer D, Chapman J, Goltsman E, Rokhsar D, Grossman AR, Nowack ECM (2016) Development of a toolbox to dissect host-endosymbiont interactions and protein trafficking in the trypanosomatid Angomonas deanei. BMC Evolutionary Biology 16 (1):247

6. Bronstein JL (2001) The exploitation of mutualisms. Ecology Letters 4 (3):277-287

7. Johnstone RA, Bshary R (2002) From parasitism to mutualism: partner control in asymmetric interactions. Ecology Letters 5 (5):634-639

8. Ferriere R, Gauduchon M, Bronstein JL (2007) Evolution and persistence of obligate mutualists and exploiters: competition for partners and evolutionary immunization. Ecology Letters 10 (2):115-126

9. Doebeli M, Hauert C (2005) Models of cooperation based on the Prisoner's Dilemma and the Snowdrift game. Ecology Letters 8 (7):748-766

10. Foster KR, Wenseleers T (2006) A general model for the evolution of mutualisms. Journal of Evolutionary Biology $19(4): 1283-1293$

11. Lee CT (2015) Inherent demographic stability in mutualist-resource-exploiter interactions. American Naturalist 185 (4):551-561

12. Shapiro JW, Turner PE (2014) The impact of transmission mode on the evolution of benefits provided by microbial symbionts. Ecology and Evolution 4 (17):3350-3361

13. Ewald PW (1987) Transmission modes and evolution of the parasitism-mutualism continuum. Annals of the New York Academy of Sciences 503:295-306

14. Douglas AE (1998) Host benefit and the evolution of specialization in symbiosis. Heredity $81: 599-603$ 
15. Herre EA, Knowlton N, Mueller UG, Rehner SA (1999) The evolution of mutualisms: exploring the paths between conflict and cooperation. Trends in Ecology \& Evolution 14 (2):4953

16. Simonsen AK, Stinchcombe JR (2014) Standing genetic variation in host preference for 530 mutualist microbial symbionts. Proceedings of the Royal Society B-Biological Sciences 281 (1797)

17. Schwartzman JA, Koch E, Heath-Heckman EAC, Zhou L, Kremer N, McFall-Ngai MJ, Ruby EG (2015) The chemistry of negotiation: Rhythmic, glycan-driven acidification in a symbiotic conversation. Proceedings of the National Academy of Sciences of the United States of America $112(2): 566-571$

18. Bull JJ, Rice WR (1991) Distinguishing mechanisms for the evolution of cooperation. Journal of Theoretical Biology 149 (1):63-74

19. Chaston J, Goodrich-Blair H (2010) Common trends in mutualism revealed by model associations between invertebrates and bacteria. FEMS Microbiology Reviews 34 (1):41-58

20. Heil M, Barajas-Barron A, Orona-Tamayo D, Wielsch N, Svatos A (2014) Partner manipulation stabilises a horizontally transmitted mutualism. Ecology Letters 17 (2):185-192

21. Heath KD, Tiffin P (2007) Context dependence in the coevolution of plant and rhizobial mutualists. Proceedings of the Royal Society B-Biological Sciences 274 (1620):1905-1912

22. Sachs JL, Mueller UG, Wilcox TP, Bull JJ (2004) The evolution of cooperation. Quarterly Review of Biology 79 (2):135-160

23. Weimer PJ (2015) Redundancy, resilience, and host specificity of the ruminal microbiota: implications for engineering improved ruminal fermentations. Frontiers in Microbiology 10 (6): 296. doi: 10.3389/fmicb.2015.00296

24. Scheuring I, Yu DW (2012) How to assemble a beneficial microbiome in three easy steps. Ecology Letters 15 (11):1300-1307

25. Rodríguez-Ruano SM, Martín-Vivaldi M, Martín-Platero AM, López-López JP, PeraltaSánchez JM, Ruiz-Rodríguez M, Soler JJ, Valdivia E, Martínez-Bueno M (2015) The hoopoe's 
uropygial gland hosts a bacterial community influenced by the living conditions of the bird. PLoS One $10(10)$

26. Soler JJ, Martín-Vivaldi M, Peralta-Sánchez JM, Ruiz-Rodríguez M (2010) Antibioticproducing bacteria as a possible defence of birds against pathogenic microorganisms. Open Ornithology Journal 3:93-100

27. Soler JJ, Martín-Vivaldi M, Ruiz-Rodríguez M, Valdivia E, Martín-Platero AM, MartínezBueno M, Peralta-Sánchez JM, Méndez M (2008) Symbiotic association between hoopoes and antibiotic-producing bacteria that live in their uropygial gland. Functional Ecology 22 (5):864871

28. Law-Brown J, Meyers PR (2003) Enterococcus phoeniculicola sp nov., a novel member of the enterococci isolated from the uropygial gland of the Red-billed Woodhoopoe, Phoeniculus purpureus International Journal of Systematic and Evolutionary Microbiology 53:683-685

29. Martín-Vivaldi M, Ruiz-Rodríguez M, Soler JJ, Peralta-Sánchez JM, Méndez M, Valdivia E, Martín-Platero AM, Martínez-Bueno M (2009) Seasonal, sexual and developmental differences in hoopoe preen gland morphology and secretions. Evidence for a role of bacteria. Journal of Avian Biology 40:191-205

30. Martín-Platero AM, Valdivia E, Ruiz-Rodríguez M, Soler JJ, Martín-Vivaldi M, Maqueda M, Martínez-Bueno M (2006) Characterization of antimicrobial substances produced by Enterococcus faecalis MRR 10-3, isolated from the uropygial gland of the hoopoe Upupa epops Applied and Environmental Microbiology 72:4245-4249

31. Martín-Vivaldi M, Peña A, Peralta-Sánchez JM, Sánchez L, Ananou S, Ruiz-Rodríguez M, Soler JJ (2010) Antimicrobial chemicals in hoopoe preen secretions are produced by symbiotic bacteria. Proceedings of the Royal Society of London, Series B Biological Sciences 277 (1678):123-130

32. Ruiz-Rodríguez M, Valdivia E, Martín-Vivaldi M, Martín-Platero AM, Martínez-Bueno M, Méndez M, Peralta-Sánchez JM, Soler JJ (2012) Antimicrobial activity and genetic profile of enteroccoci isolated from hoopoes uropygial gland. PLoS One 7 e41843: 41841-41846 

Bueno M (2009) Symbiotic bacteria living in the hoopoe's uropygial gland prevent feather degradation. Journal of Experimental Biology 212 (22):3621-3626

34. Martín-Vivaldi M, Soler JJ, Peralta-Sánchez JM, Arco L, Martín-Platero AM, Martínez-Bueno

M, Ruiz-Rodríguez M, Valdivia E (2014) Special structures of hoopoe eggshells enhance the adhesion of symbiont-carrying uropygial secretion that increase hatching success. Journal of Animal Ecology 83 (6):1289-1301

35. Soler JJ, Martín-Vivaldi M, Peralta-Sánchez JM, Arco L, Juárez-García-Pelayo N (2014) Hoopoes color their eggs with antimicrobial uropygial secretions. Naturwissenschaften 101 (9):697-705

36. Martínez-García A, Martín-Vivaldi M, Ruiz-Rodríguez M, Martínez-Bueno M, Arco L, Rodríguez-Ruano S, Peralta-Sánchez JM, Soler JJ (2016) The microbiome of the uropygial secretion in hoopoes is shaped along the nesting phase. Microbial Ecology 72 (1):252-261 doi: $10.1007 / \mathrm{s} 00248-016-0765-1$

37. Ruiz-Rodríguez M, Soler JJ, Martín-Vivaldi M, Martín-Platero AM, Méndez M, PeraltaSánchez JM, Ananou S, Valdivia E, Martínez-Bueno M (2014) Environmental factors shape the community of symbionts in the hoopoe uropygial gland more than genetic factors. Applied and Environmental Microbiology 80 (21):6714-6723

38. Ruiz-Rodríguez M, Martínez-Bueno M, Martín-Vivaldi M, Valdivia E, Soler JJ (2013) Bacteriocins with a broader antimicrobial spectrum prevail in enterococcal symbionts isolated from the hoopoe's uropygial gland. FEMS Microbiology Ecology 85 (3):495-502

39. Hammond AC (1995) Leucaena toxicosis and its control in ruminants. Journal of Animal Science 73 (5):1487-1492

40. Giraudeau M, Czirják GÁ, Duval C, Bretagnolle V, Gutierrez C, Guillon N, Heeb P (2013) Effect of preen oil on plumage bacteria: An experimental test with the mallard. Behavioural Processes 92:1-5 doi:_10.1016/j.beproc.2012.08.001 
41. Giraudeau M, Duval C, Guillon N, Bretagnolle V, Gutierrez C, Heeb P (2010) Effects of access to preen gland secretions on mallard plumage. Naturwissenschaften 97 (6):577-581. doi:10.1007/s00114-010-0673-z

42. Czirják GÁ, Pap PL, Vágási CI, Giraudeau M, Mureşan C, Mirleau P, Heeb P (2013) Preen gland removal increases plumage bacterial load but not that of feather-degrading bacteria. Naturwissenschaften 100 (2):145-151. doi:10.1007/s00114-012-1005-2

43. Hird SM (2017) Evolutionary biology needs wild microbiomes. Frontiers in Microbiology $8: 725$

44. Martínez-García A, Soler JJ, Rodríguez-Ruano SM, Martínez-Bueno M, Martín-Platero AM, Juárez-García-Pelayo N, Martín-Vivaldi M (2015) Preening as a vehicle for key bacteria in hoopoes. Microbial Ecology 70 (4):1024-1033

45. Rodríguez-Ruano S. (2015) Diversidad bacteriana en la glándula uropigial de la abubilla: Dinámica estacional y beneficios asociados. PhD Thesis, Universidad de Granada.

46. Soler JJ, Martínez-García A, Rodríguez-Ruano S, Martínez-Bueno M, Martín-Platero AM, Peralta-Sánchez JM, Martín-Vivaldi M (2016) Nestedness of hoopoes' bacterial communities: symbionts from the uropygial gland to the eggshell. Biological Journal of the Linnean Society 118 (4):763-773 doi: 10.1111/bij.12772

47. Martínez-García A, Martín-Vivaldi M, Rodríguez-Ruano S, Peralta-Sánchez JM, Valdivia E, Soler Juan J (2016) Nest bacterial environment affects microbiome of hoopoe eggshells, but not that of the uropygial secretion PLoS One 11 (7). doi:10.1371/journal.pone.0158158

48. Benjamini Y, Hochberg Y (1995) Controlling the false discovery rate: a practical and powerful approach to multiple testing. Journal of the Royal Statistical Society Series B (Methodological) 57 (1):289-300.

49. Vautrin E, Vavre, F (2009) Interactions between vertically transmitted symbionts: cooperation or conflict? Trends in Microbiology 17 (3):95-99 
50. Braun MS, Zimmermann S, Danner M, Rashid Ho, Wink M (2016) Corynebacterium uropygiale sp. nov., isolated from the preen gland of Turkeys (Meleagris gallopavo). Systematic and Applied Microbiology 39 (2):88-92

51. Law-Brown J (2001) Chemical defence in the red-billed woodhoopoe, Phoeniculus purpureus. University of Cape Town, Cape Town.

52. Burger BV, Reiter B, Borzyk O, Du Plessis MA (2004) Avian exocrine secretions. I. Chemical characterization of the volatile fraction of the uropygial secretion of the green woodhoopoe, Phoeniculus purpureus. Journal of Chemical Ecology 30 (8):1603-1611

53. Moran NA, Wernegreen JJ (2000) Lifestyle evolution in symbiotic bacteria: insights from genomics. Trends in Ecology \& Evolution 15 (8):321-326

54. Mandel MJ (2010) Models and approaches to dissect host-symbiont specificity. Trends in Microbiology 18 (11):504-511

55. Cafaro MJ, Poulsen M, Little AEF, Price SL, Gerardo NM, Wong B, Stuart AE, Larget B, Abbot P, Currie CR (2011) Specificity in the symbiotic association between fungus-growing ants and protective Pseudonocardia bacteria. Proceedings of the Royal Society B-Biological Sciences 278 (1713):1814-1822

56. Andersen SB, Hansen LH, Sapountzis P, Sorensen SJ, Boomsma JJ (2013) Specificity and stability of the Acromyrmex-Pseudonocardia symbiosis. Molecular Ecology 22 (16):4307-4321 
Table 1. Results of a Repeated Measures ANOVA analyzing the effect of the inoculation of uropygial secretion, collected from their own gland or from a nestling of a different nest (nestling type), on the number of Operational Taxonomic Units (OTUs) detected in nestling secretions before and one week after inoculation ( $R$ : repeated measures). The model also included the effect of year and the random effect of nest identity nested within study year. Significant effects are marked in bold.

\begin{tabular}{llccc}
\hline & & df & F & $p$ \\
\hline R1 & & & & \\
R1 x Nestling Type & Fixed & 1 & 3.27 & 0.088 \\
R1 x Year & Fixed & 1 & 7.46 & $\mathbf{0 . 0 1 1}$ \\
R1 x Nest (Year) & Rixed & 1 & 0.07 & 0.796 \\
Error & & 16 & 1.45 & 0.227 \\
& & 17 & & \\
\hline
\end{tabular}


Table 2. Effects of the covering of female glands (Female treatment) on the similarities between mothers and their nestlings (repeated measure R1) in the composition of the core microbiome of their uropygial secretions. Females' samples were collected before the experiment covering their glands and those of nestlings when they were 16 days old. Each repeated factor (R1) compares PCo scores of nestling and female. Results for R1 did not qualitatively change when not including the interaction with female treatment in the model. DFs adjusted to the number of nests. Significant $p$-values are in bold.

df $F$

$\begin{array}{llrrr}\text { PCo1 } & & & & \\ \text { R1 } & \text { Fixed } & 1 & 4.04 & 0.062 \\ \text { R1 x Female treatment } & \text { Fixed } & 1 & 1.55 & 0.223 \\ \text { Error } & & 16 & & \\ & & & & \\ \text { PCo2 } & & & & \\ \text { R1 } & \text { Fixed } & 1 & 112.85 & <\mathbf{0 . 0 0 0 1} \\ \text { R1 x Female treatment } & \text { Fixed } & 1 & 5.65 & \mathbf{0 . 0 3 0} \\ \text { Error } & & 16 & & \\ \end{array}$


Table 3. Results from Repeated measures ANOVAs exploring the effects of the experimental inoculation of nestlings glands with own uropygial secretion or that of a foreign nestling (nestling type) on the change of the microbiome composition after the inoculation experiment (repeated measure R1). Each repeated factor (R1) compares PCo scores of nestlings before and after inoculation. Results for R1 did not change when not including the interaction with type of nestling in the model. Significant $\mathrm{p}$-values are in bold.

\begin{tabular}{|c|c|c|c|c|}
\hline & & df & $\mathrm{F}$ & $p$ \\
\hline \multicolumn{5}{|l|}{ PCo1 } \\
\hline $\mathrm{R} 1$ & Fixed & 1 & 11.46 & 0.002 \\
\hline R1 x Nestling type & Fixed & 1 & 4.92 & 0.033 \\
\hline Error & & 34 & & \\
\hline \multicolumn{5}{|l|}{ PCo2 } \\
\hline R1 & Fixed & 1 & 1.61 & 0.214 \\
\hline R1 x Nestling type & Fixed & 1 & 5.02 & 0.032 \\
\hline Error & & 34 & & \\
\hline \multicolumn{5}{|l|}{ PCo3 } \\
\hline R1 & Fixed & 1 & 5.87 & 0.021 \\
\hline R1 $x$ Nestling type & Fixed & 1 & 3.90 & 0.056 \\
\hline Error & & 34 & & \\
\hline
\end{tabular}


Table 4. Results of a General Linear Model (GLM) exploring the association between density of cultivable bacteria in nestling secretions (dependent variable, $(\mathrm{cfu} / \mu \mathrm{l}))$ and that of females. Whether or not uropygial gland of females was experimentally covered (female treatment) as well as its interaction with the bacterial density of female secretion were included in the model $\left(1^{*} 2\right)$. The main effects remained statistically significant when removing the interaction. Degrees of freedom were adjusted to number of nests. Significant $p$-values are in bold.

$\begin{array}{ccc}\text { df } & F & p \\ 1 & 8.99 & \mathbf{0 . 0 1 1} \\ 1 & 5.30 & \mathbf{0 . 0 3 8} \\ 1 & 3.91 & 0.069 \\ 12 & & \end{array}$

663

1) Female treatment

2) $\log (\mathrm{CFUs} / \mu \mathrm{l})$ female secretion

3) $1 * 2$

12

p 
Table 5. Summary of effects for the Operational Taxonomic Units (OTUs) with significant contributions to properties of the microbiome of hoopoe nestling secretions (see analyses in Supplemental Material-II).

\begin{tabular}{|c|c|c|c|c|c|}
\hline OTU & $\begin{array}{l}\text { Affected by } \\
\text { covering } \\
\text { female gland }\end{array}$ & $\begin{array}{l}\text { Related with } \\
\text { presence in } \\
\text { female gland }\end{array}$ & $\begin{array}{l}\text { Effect of } \\
\text { presence in } \\
\text { donor }\end{array}$ & $\begin{array}{l}\text { Relation with } \\
\text { TSA growing }\end{array}$ & Probable source \\
\hline 242 & & & Yes & Negative & External \\
\hline 306 & Positive & & Yes & Positive & Gut \\
\hline 346 & Negative & & Yes & Negative & Female gland \\
\hline 406 & Positive & & Yes & & External/Gut \\
\hline 466 & Negative & Yes $* \star$ & Yes & & Female gland \\
\hline 566 & & Yes & Yes & Negative & Gut \\
\hline
\end{tabular}

** Present in all females except in one experimental 
Fig. 1 Schematic representation of the design and steps used for the two experiments. a) Experiment 1: manipulation of female access to the uropygial gland, to test the direct transmission

Fig. 2 Effect of the experiment inoculating uropygial glands of hoopoe nestlings with secretions from the same nestling (control) or from a nestling of a different nest (experimental) on richness of the bacterial community hosted in their gland

Fig. 3 Prevalence of Operational Taxonomic Units (OTUs) found in uropygial secretions of nestling hoopoes before (black bars) and a week after (white bars) the inoculation of their glands with (a) their own secretion or (b) the secretion from a nestling of a different nest

Fig. 4 (a) Influence of manipulating females' access to their uropygial gland on the differences between hoopoe females and their nestlings in PCo 2 scores. Means and 95\% confidence intervals calculated for the number of nests are presented. (b) Differences in the composition of the hoopoe uropygial gland core microbiome, among control, experimental females and their nestlings, as reflected for PCo1 and PCo2

Fig. 5 PCo plot representing, by their proximity, the resemblance in composition of the microbiome among samples of uropygial secretions from nestlings before (initial) and a week after (final) the inoculation experiment. Control nestlings were inoculated their own secretion, while another nestling from each nest (cross-inoculated) was inoculated with secretion taken from a different brood

Fig. 6 Effects of manipulating female's access to their uropygial gland on (a) the abundance of cultivable bacteria in nestling secretions and (b) the relationship between the bacterial growth from female and nestling secretions 
Fig 1

a

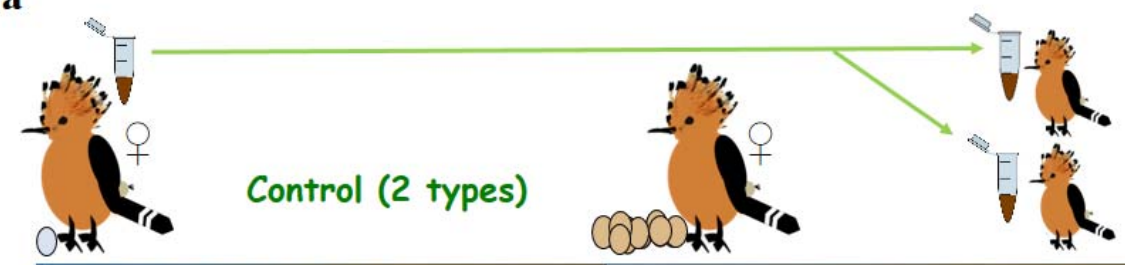

Nestling period
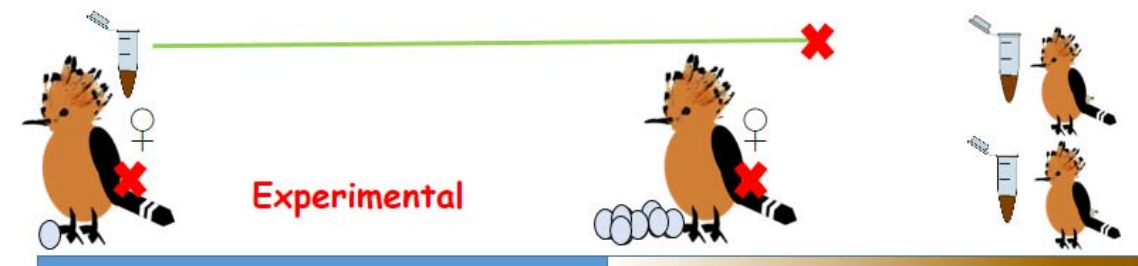

Incubation

Nestling period

Day 1:

$\uparrow$

- Sampling

Day 16:

- Female manipulation

- Nestling sampling

706

b

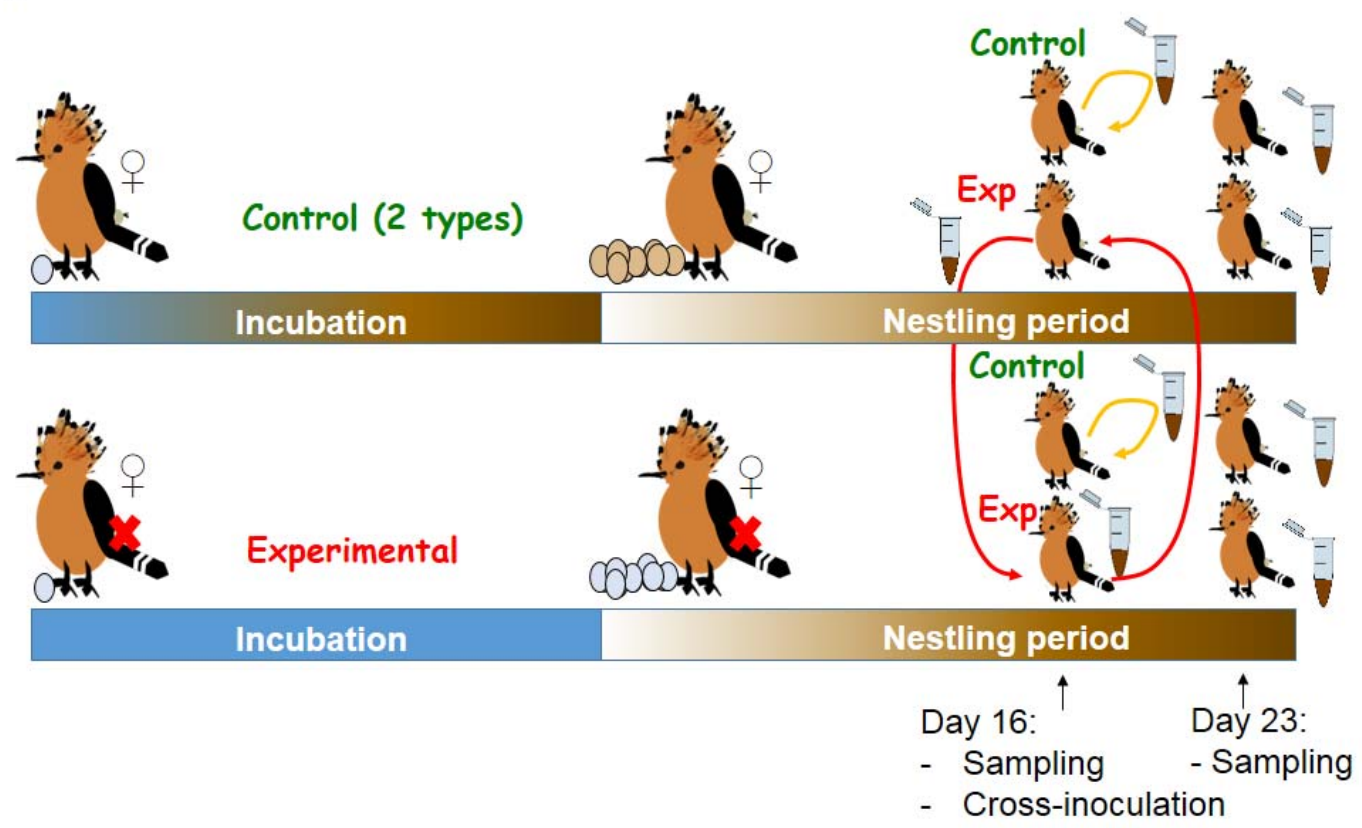


Fig. 2

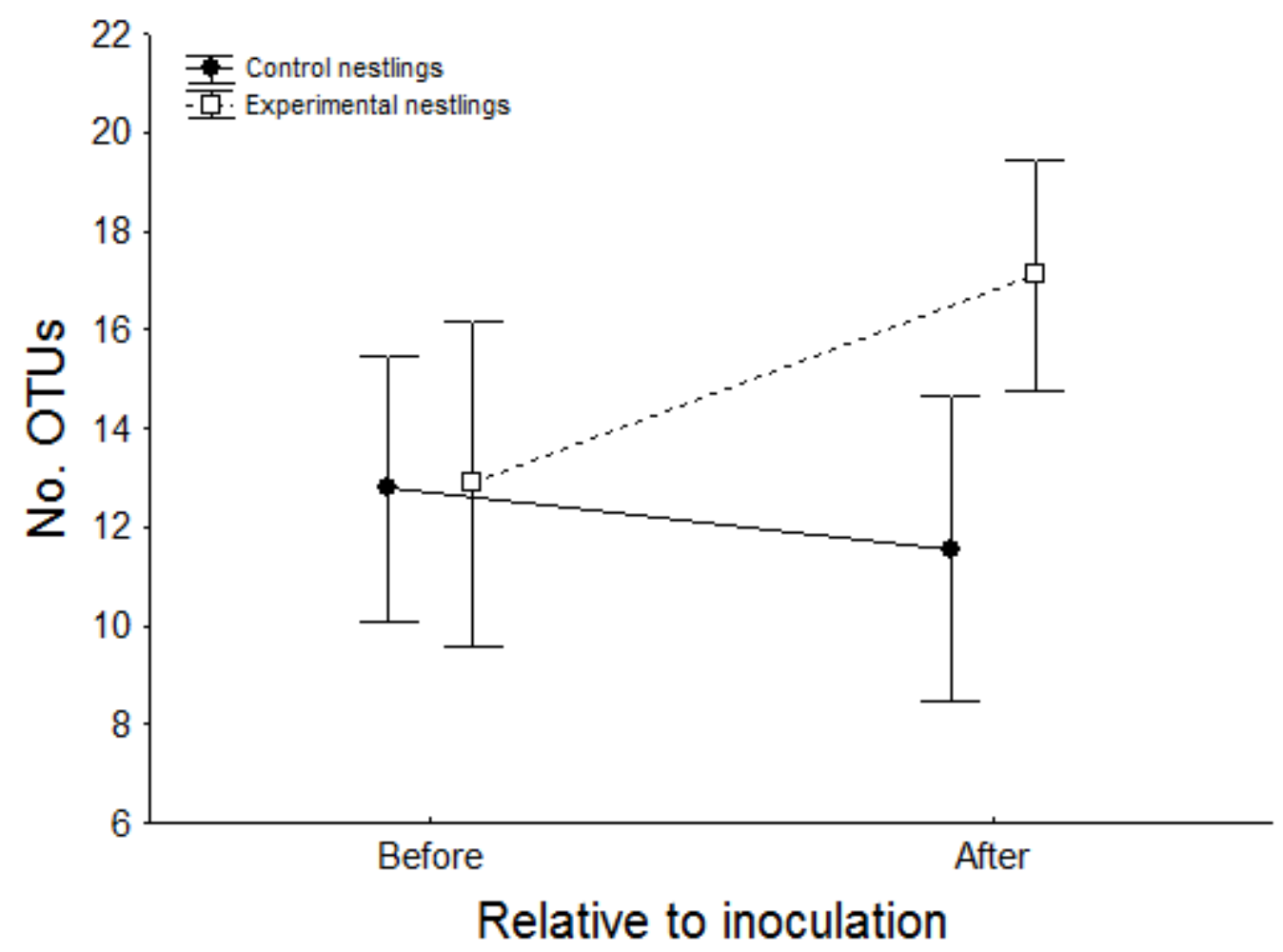


Fig 3.

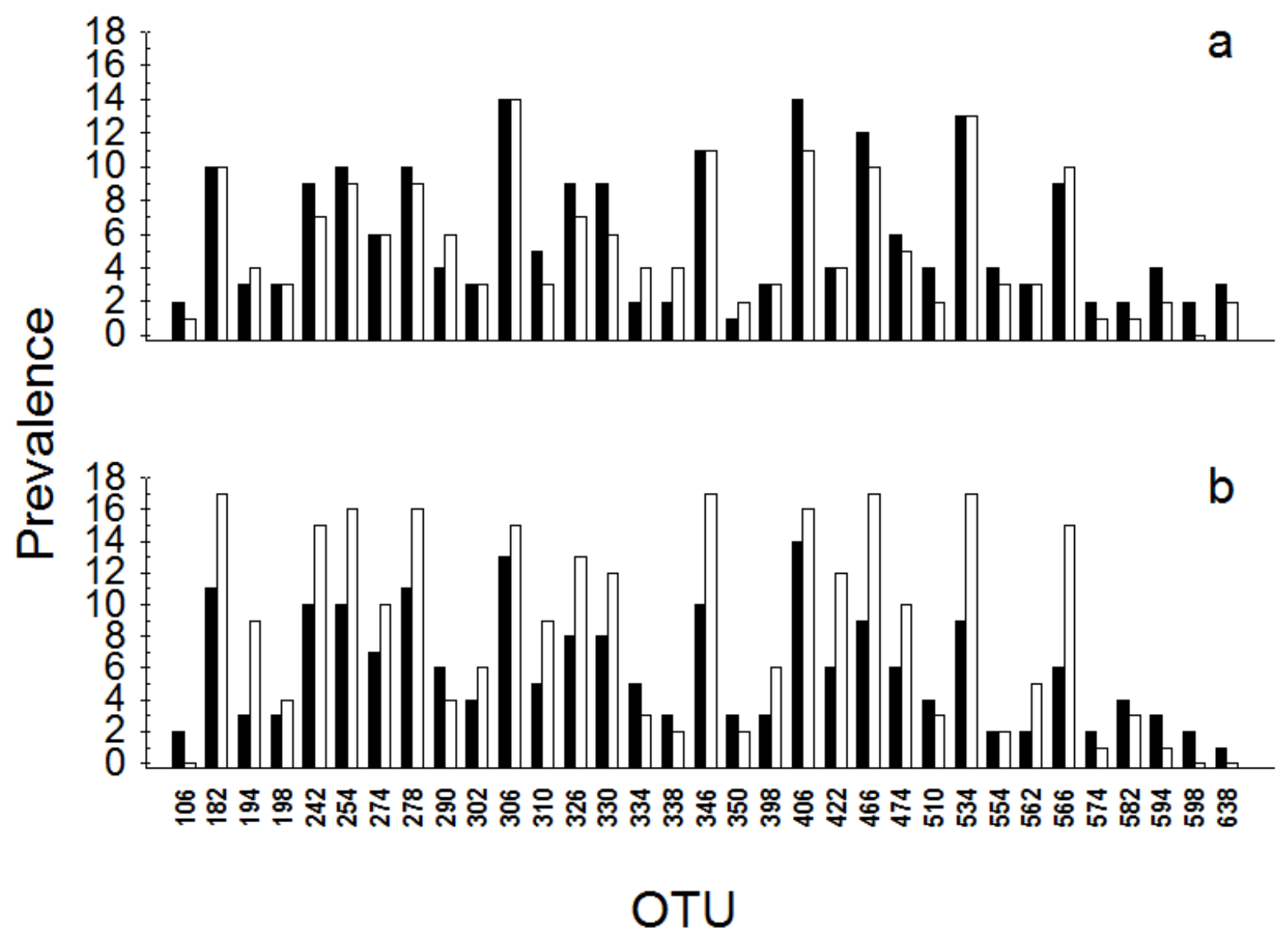


Fig. 4a

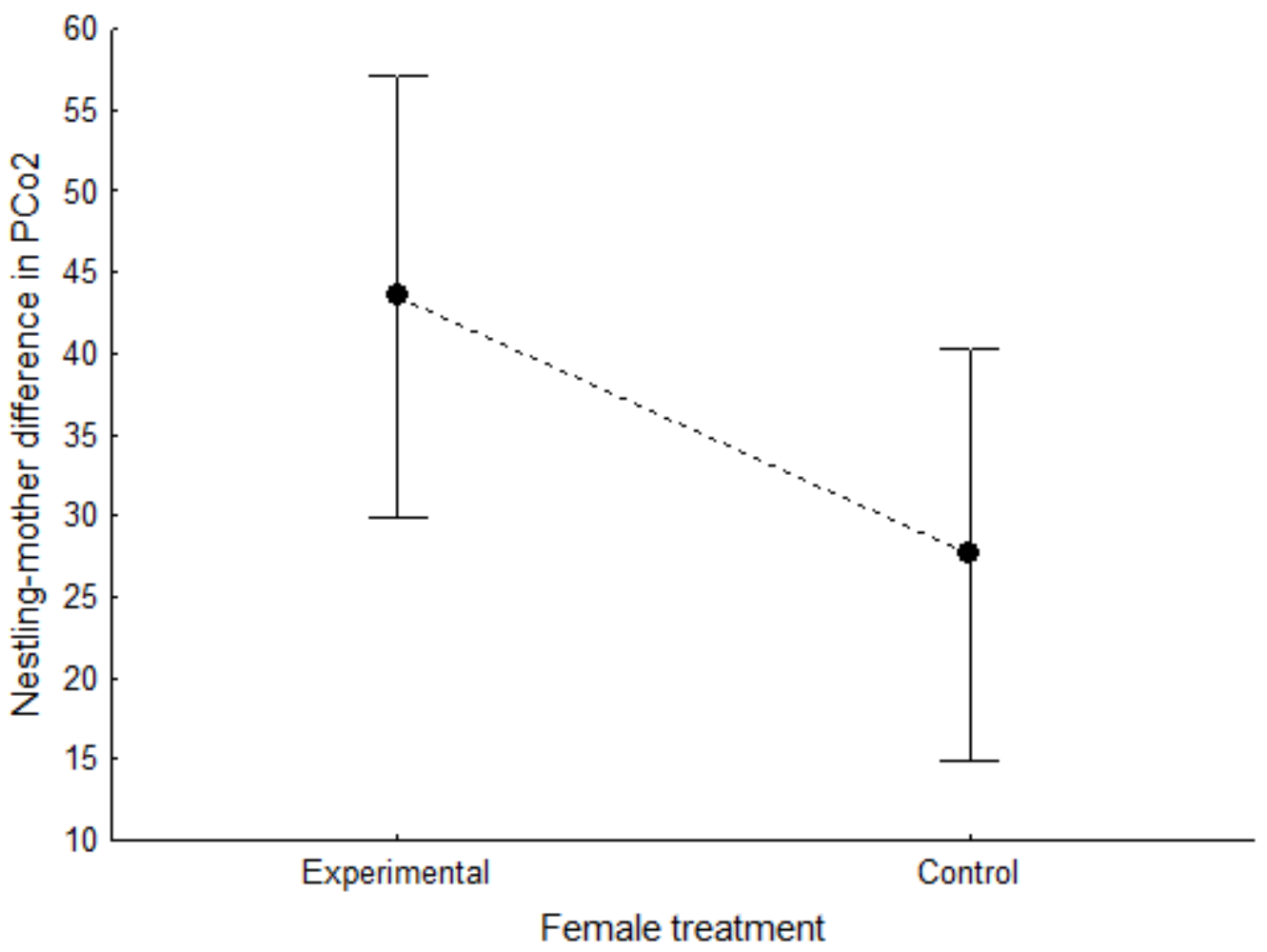

Fig 4b

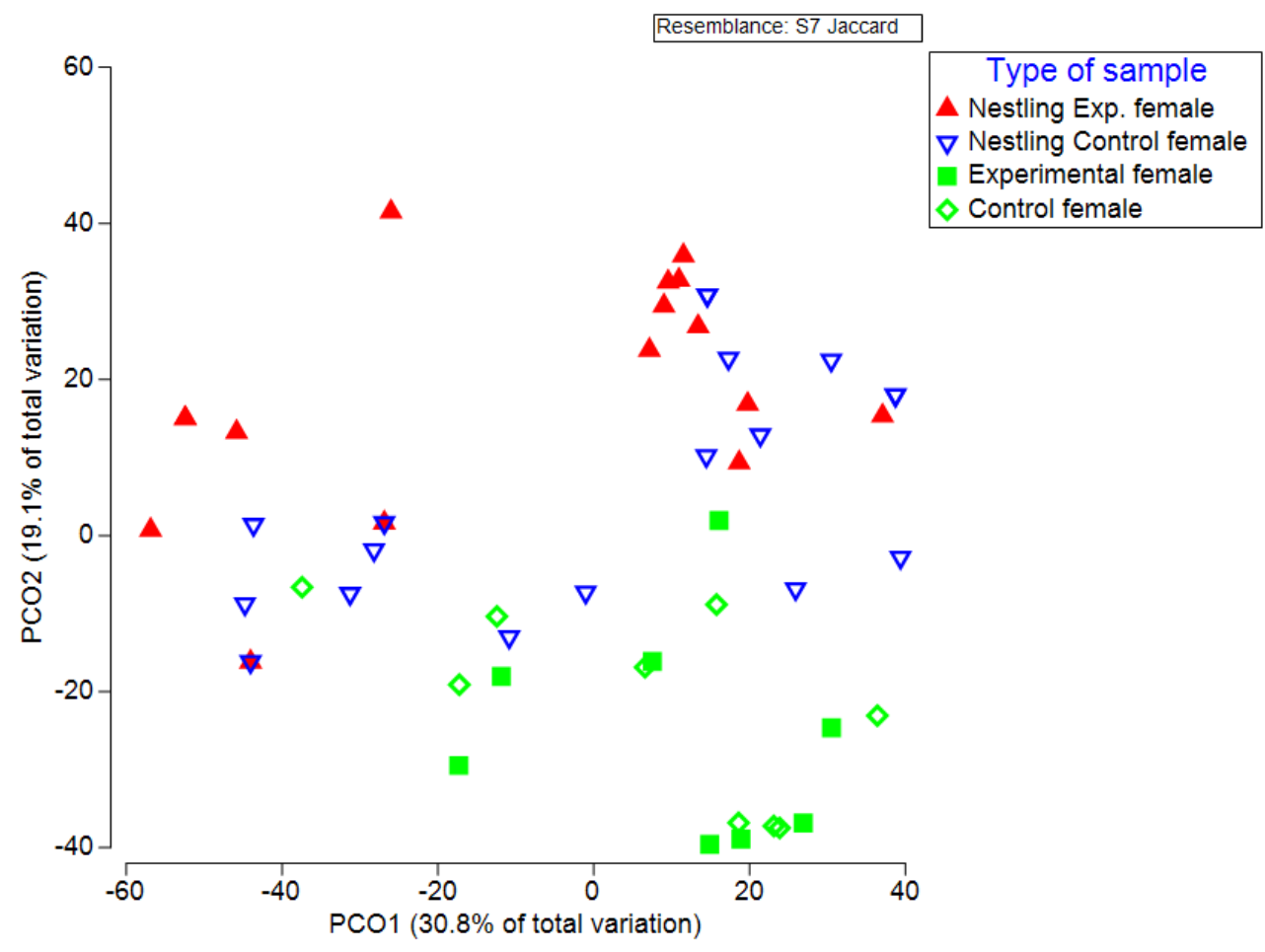


Fig 5

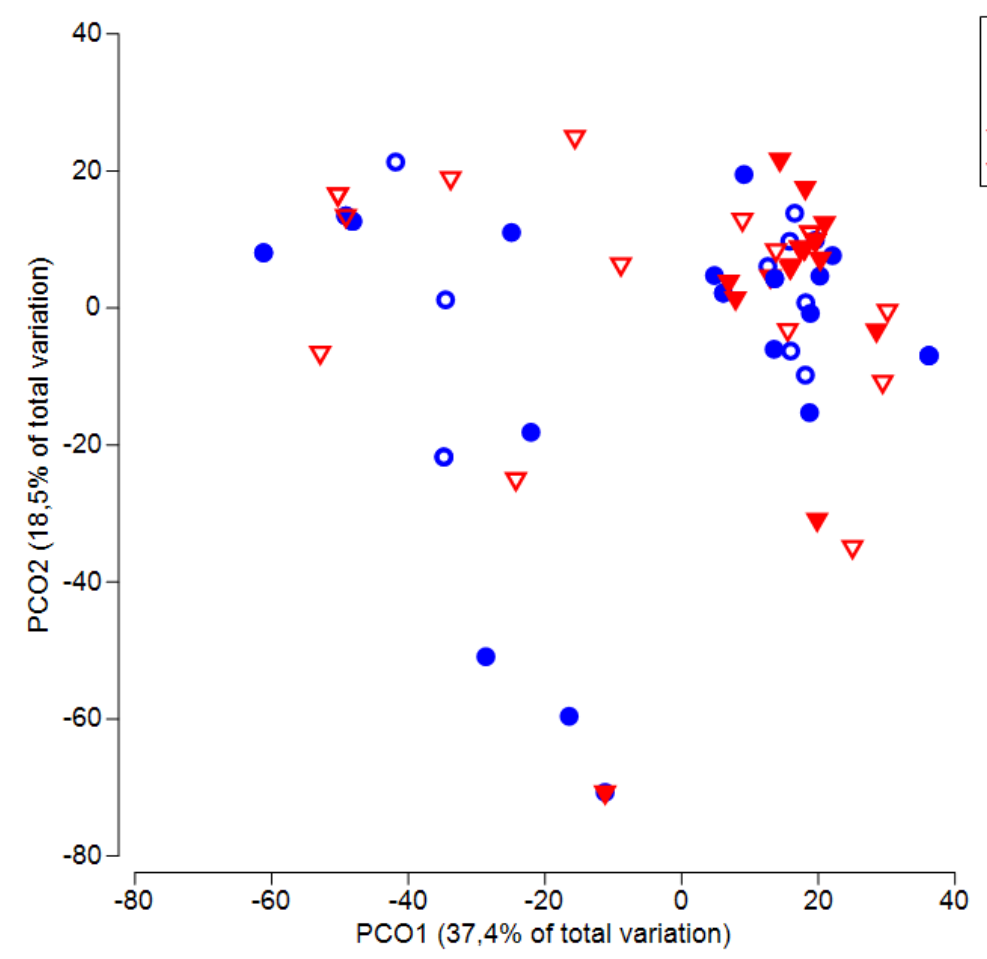


Fig. 6a

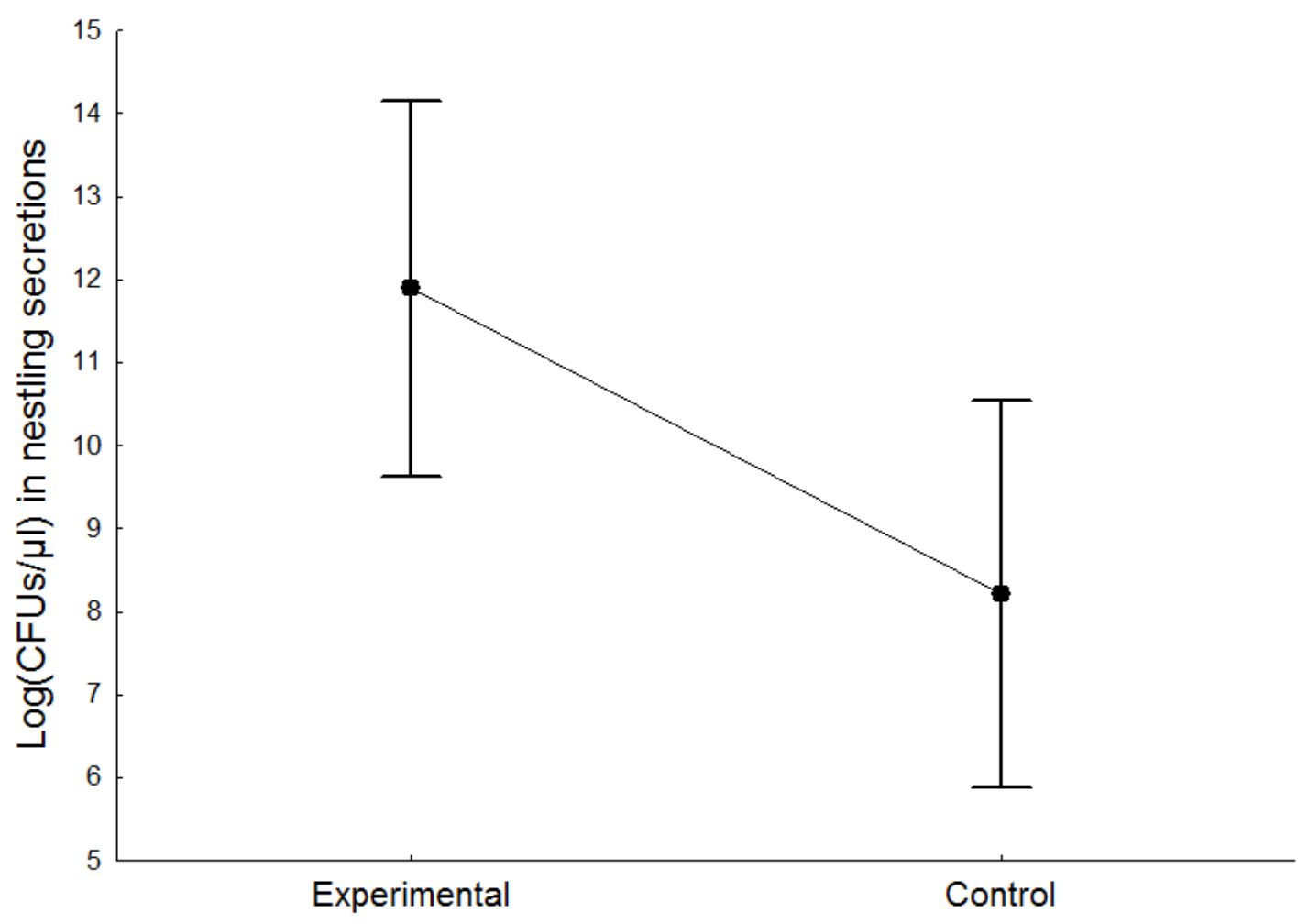

Female treatment

Fig 6b

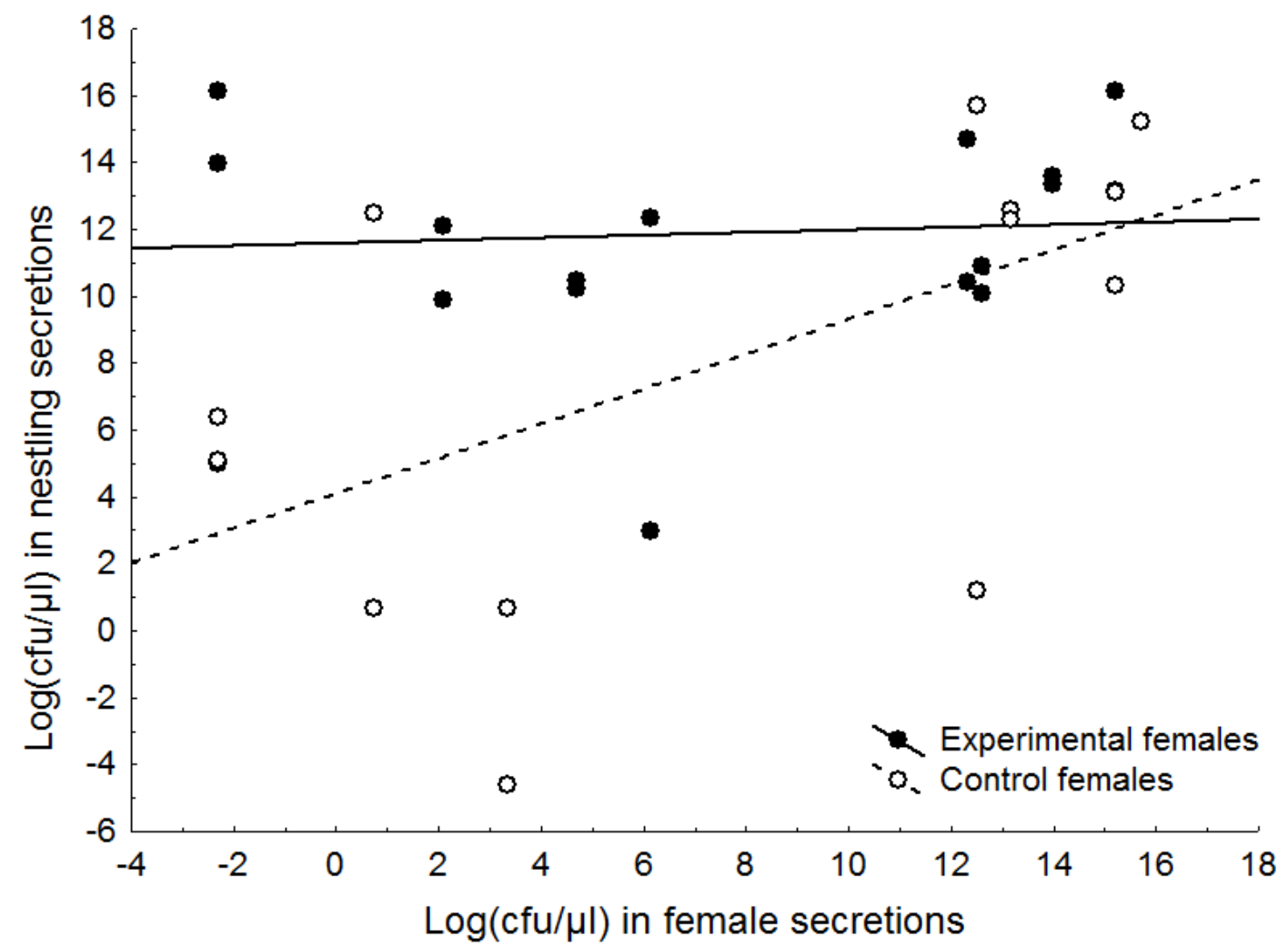




\section{Acquisition of uropygial gland microbiome by hoopoe nestlings}

\section{Microbial Ecology}

737 M. Martín-Vivaldi ${ }^{\mathrm{a}, \mathrm{b} *}$, J. J. Soler, Ángela Martínez-García, L. Arco, N. Juárez-García-Pelayo, M. Ruiz738 Rodríguez, M. Martínez-Bueno

739 * Correspondence author. e-mail: $\underline{\operatorname{mmv} @ u g r . e s}$

740 a) Departamento de Zoología, Universidad de Granada, E-18071 Granada, Spain.

741 b) Estación Experimental de Zonas Áridas (CSIC) E-04120 Almería, Spain.

742

743 SUPPLEMENTAL MATERIAL -I. ARISA methodology

744

745 ARISA (Automated rRNA Intergenic Spacer Analysis, Fisher \& Triplett 1999) amplifies an intergenic 746 transcribed spacer (ITS) region between the prokaryotic $16 \mathrm{~S}$ and $23 \mathrm{~S}$ rDNA. The ITS was amplified using 747 the primer pair ITSF (5'-GTCGTAACAAGGTAGCCGTA-3') and ITSReub (5'-

748 GCCAAGGCATCCACC-3') (Cardinale et al. 2004). The primer ITSReub was labelled fluorescently with

749 6-FAM. Amplifications were performed in $50 \mu 1$ reaction volumes containing ultrapure H2O, 1x 5 PRIME 750 MasterMix including 1.5 mM Magnesium, $200 \mathrm{mM}$ dNTPs, 1.25 U Taq polymerase (5 PRIME, Hamburg, 751 Germany), $0.2 \mathrm{mM}$ of primers and $5 \mu 1$ of diluted DNA 1:10. PCRs were carried out in Eppendorf 752 Mastercycler Nexus Family. Fragments were amplified under the following conditions: initial denaturation 753 at $94{ }^{\circ} \mathrm{C} 2 \mathrm{~min}$, followed by 30 cycles with denaturation at $94{ }^{\circ} \mathrm{C} 45 \mathrm{~s}$, annealing at $52{ }^{\circ} \mathrm{C} 45 \mathrm{~s}$ and 754 extension at $72{ }^{\circ} \mathrm{C} 1 \mathrm{~min}$, with a final extension at $72{ }^{\circ} \mathrm{C} 5 \mathrm{~min}$. Amplified PCR products were diluted 1:10 755 and denatured by heating in formamide. Fragment lengths were determined by mean of automated 756 fluorescent capillary electrophoresis on 3130 Genetic Analyzer. Electropherogram peak values were 757 calculated after interpolation with an internal size standard named GeneScan ${ }^{\mathrm{TM}} 1200$ LIZ dye Size 758 Standard (both Applied Biosystems). 

different bacterial Operational Taxonomic Units (OTUs) within each sample. Scripts in R-environment [http://cran.r-project.org/]) available at http://www.ecology-research.com, were used for binning DNA

762 fragment lengths from different samples. Binning exercise was performed by establishing a window size of 4 pair of bases and a distance of two consecutive binning frames (i.e. shift) of 0.1 . Only peaks with values of relative intensity of fluorescence larger than $0.09 \%$ and fragments above a threshold of 50 fluorescence units that ranged between 100 and 1,000 bp (Ramette 2009) have been considered. Molecular fingerprinting techniques are highly reproducible, robust, and have been proven useful for comparative 767 analysis of microbial community structure (Loisel et al. 2006; Bent \& Forney 2008).

\section{References}

Bent, S.J. \& Forney, L.J. (2008). The tragedy of the uncommon: understanding limitations in the analysis of microbial diversity. ISME Journal, 2, 689-695. doi: 10.1038/ismej.2008.44

Cardinale, M., Brusetti, L., Quatrini, P., Borin, S., Puglia, A., Rizzi, A., Zanardini, E., Sorlini, C., Corselli, C. \& Daffonchio, D. (2004). Comparison of different primer sets for use in automated ribosomal intergenic spacer analysis of complex bacterial communities. Applied and Environmental Microbiology, 70, 6147-6156. doi: 10.1128/AEM.70.10.6147-6156.2004

Fisher, M.M. \& Triplett, E.W. (1999). Automated approach for ribosomal intergenic spacer analysis of microbial diversity and its application to freshwater bacterial communities. Applied Environmental Microbiology, 65, 4630-4636.

Loisel, P., Harmand, J., Zemb, O., Latrille, E., Lobry, C., Delgenès, J.P. \& Godon, J.J. (2006). Denaturing gradient electrophoresis (DGE) and single-strand conformation polymorphism (SSCP) molecular fingerprintings revisited by simulation and used as a tool to measure microbial diversity. Environmental Microbiology, 8, 720-731. doi: 10.1111/j.1462-2920.2005.00950.x

Ramette, A. (2009). Quantitative community fingerprinting methods for estimating the abundance of operational taxonomic units in natural microbial communities. Applied and Environmental Microbiolology, 75, 2495-2505. doi: 10.1128/AEM.02409-08 


\section{Microbial Ecology}

788 M. Martín-Vivaldia,b*, J. J. Soler, Ángela Martínez-García, L. Arco, N. Juárez-García-Pelayo, M. Ruiz789 Rodríguez, M. Martínez-Bueno

790* Correspondence author. e-mail:mmv@ugr.es

791 a) Departamento de Zoología, Universidad de Granada, E-18071 Granada, Spain.

792 b) Estación Experimental de Zonas Áridas (CSIC) E-04120 Almería, Spain.

793

794 SUPPLEMENTAL MATERIAL-II. Importance of particular OTUs 795

796 In order to know which of the OTUs detected in secretions by ARISA analyses were responsible of the 797 detected experimental effects on cultivable bacterial load and composition of bacterial communities, we 798 explored the association of the presence of each OTU of the core microbiome with these effects.

799

\section{Relationship with cultivable bacterial load}

801 The best GLZ models (those with lower AIC values differing in less than two unities) explaining bacterial 802 load of secretions (considering all secretions from nestlings and females) included combinations of eight 803 OTUs (Table 1) with positive and negative associations. The more clear association was detected for the 804 OTU306 (Fig. 1) suggesting that it is the main component of the microbiome of hoopoe uropygial secretions 805 able of growing in TSA medium in aerobic conditions. The detection of both positive and negative 806 associations between presence of some OTUs and cultivable bacterial density may be the consequence of 807 direct antagonistic effects or competence between both groups of OTUs.

Table 1. Best subsets of OTUs present in hoopoe uropygial secretions explaining bacterial growth in TSA general medium in a GLZ model with logit link function and a normal distribution.

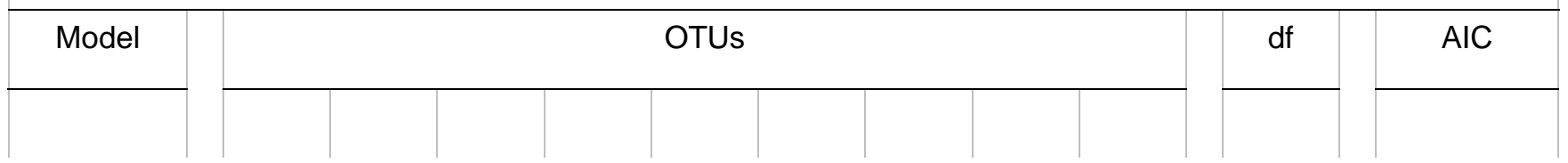




\begin{tabular}{|c|c|c|c|c|c|c|c|c|c|c|c|}
\hline 1 & 242 & 254 & 306 & 346 & & & & & & 4 & 578,1685 \\
\hline 2 & 242 & & 306 & 346 & & 422 & & 534 & 566 & 6 & 578,8353 \\
\hline 3 & 242 & 254 & 306 & 346 & & 422 & & 534 & 566 & 7 & 578,8357 \\
\hline 4 & 242 & 254 & 306 & 346 & & & & & 566 & 5 & 578,9262 \\
\hline 5 & 242 & 254 & 306 & 346 & 406 & & & & & 5 & 579,0754 \\
\hline 6 & 242 & 254 & 306 & 346 & & & & 534 & 566 & 6 & 579,1720 \\
\hline 7 & & & 306 & 346 & & 422 & & 534 & 566 & 5 & 579,2048 \\
\hline 8 & 242 & 254 & 306 & 346 & & & 466 & & & 5 & 579,4899 \\
\hline 9 & & & 306 & 346 & & & & 534 & 566 & 4 & 579,5372 \\
\hline 10 & 242 & & 306 & & & 422 & & 534 & 566 & 5 & 579,5399 \\
\hline & & & & & & & & & & & \\
\hline
\end{tabular}

808

809

810

811

812

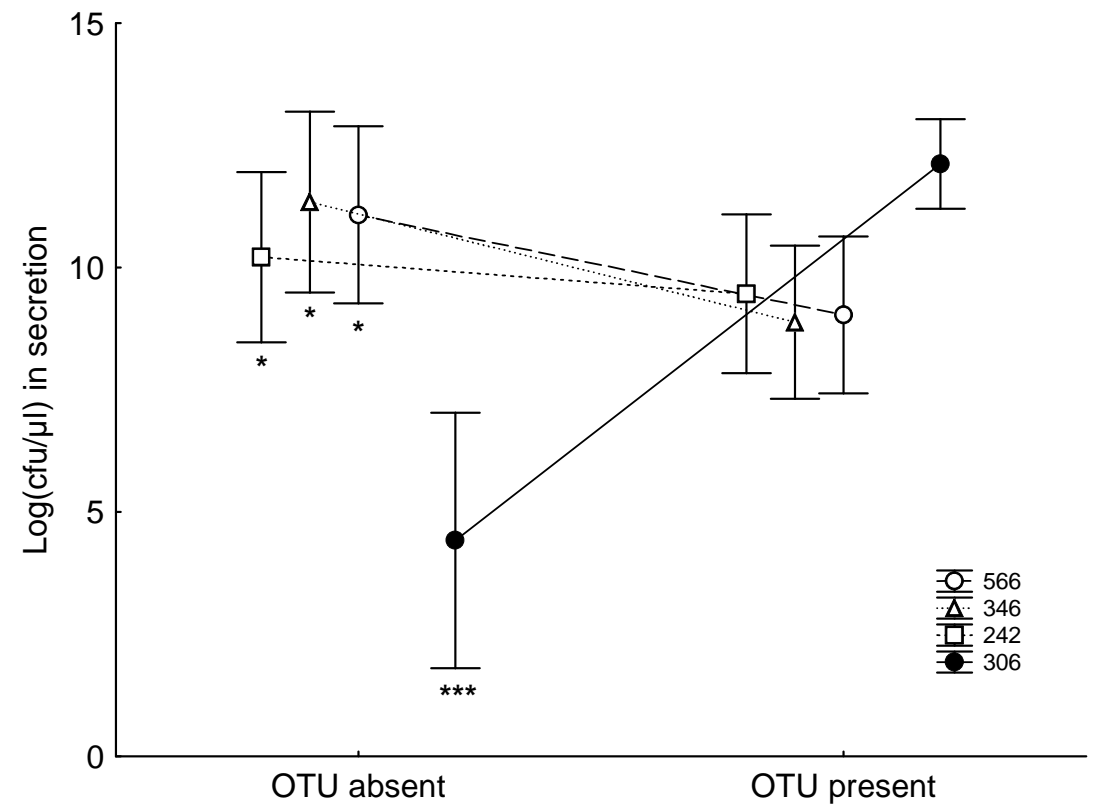

813

814 Figure 1. Relationship between the presence of OTUs in hoopoe secretions and their cultivable bacterial load. 815 Only the OTUs from the best subsets in table 2 with a significant Wald value in the whole effects model (* $816 p<0,05, * * * p<0,001)$ are presented. Whiskers show marginal means and $95 \%$ confident intervals

817

818

819 


\section{Influence of female microbiome on nestling microbiome}

821

822 The effect of covering female glands on the similarity of the microbiomes of nestlings and females was 823 specially associated to the presence in secretions of three OTUs. Two of them correlated to the space 824 occupied by the microbiomes of nestlings of experimental females (OTU306 and OTU406, Fig. 2) and 825 another correlated to the position of the microbiomes of nestlings of control females (OTU466, Fig. 2). In 826 accordance with this interpretation, the presence of these three OTUs in nestlings was affected by 827 manipulation of female access to its gland (Table 2). In addition, prevalence of the OTU346 in nestlings was 828 significantly associated with female experimental treatments when considering only females with the OTU 829 in their glands $(\mathrm{GLZ}, \mathrm{Wald}=4.78, \mathrm{p}=0.029, \mathrm{q}=0.046)$. For two additional OTUs (OTU466 and OTU346) 830 the manipulation of female access to their glands caused a reduction in the percentage of nestlings that 831 harbored the OTU (Fig. 3). On the other hand, two OTUs (306 and 406) were more frequent in nestlings 832 from experimental than from control females (Fig. 3).

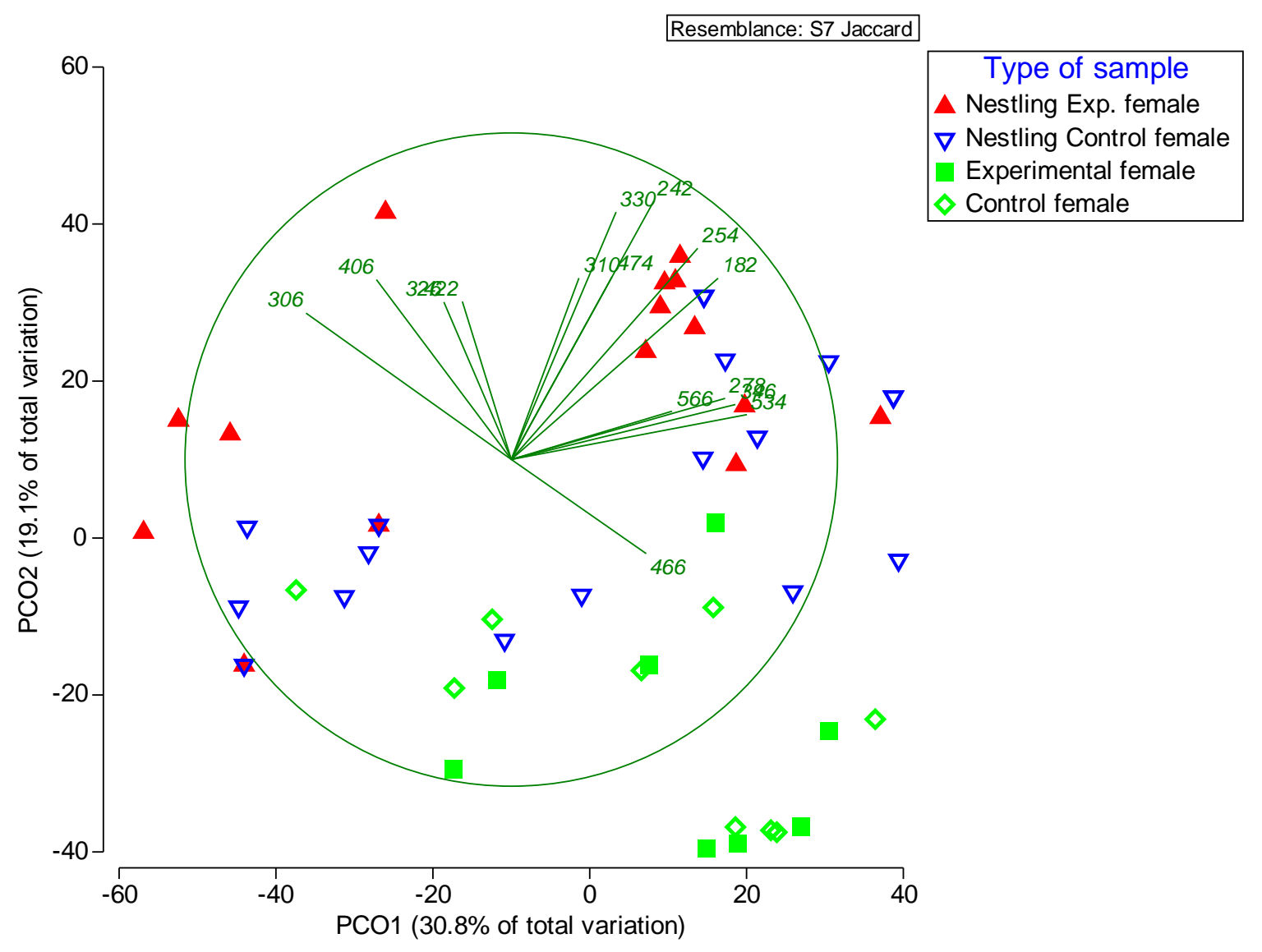

Figure 2. PCO plot showing the resemblance between samples of the experiment manipulating female access 
to its gland. The influence of each of the OTUs of the core microbiome is represented by the green lines being their length the value of the Spearman correlation coefficient.

833

834 
Table 2. Influence of (1) the presence of an OTU in female secretions and (2) the experimental manipulation of female access to its gland (female treatment), on the occurrence of the same OTU in nestlings uropygial gland secretions. The table shows the results only for the four OTUs of the core microbiome with a significant effect of these factors. The best models according to the AIC criterion for each OTU in a GLZ design with both factors as predictors are presented. We applied the false discovery rate correction for multiple tests (the 14 tests for the OTUs of the core microbiome present in females) to calculate the q-values (adjusted p-values). The factors of each model with a significant effect are indicated in bold.

\begin{tabular}{lcccccccc}
\hline OTU & Model & $\begin{array}{c}\text { OTU } \\
\text { presence } \\
\text { in female }\end{array}$ & $\begin{array}{c}\text { Female } \\
\text { treatment }\end{array}$ & df & AIC & $\begin{array}{c}\text { L.Ratio } \\
\text { Chi'/Wald }\end{array}$ & $\mathbf{p}$ & q-value \\
\hline 306 & 1 & + & + & 2 & 35,49 & 9,81 & 0,007 & $\mathbf{0 . 0 3 0}$ \\
& 2 & & + & 1 & 36,21 & 7,09 & 0,008 & $\mathbf{0 . 0 3 4}$ \\
\hline 406 & 1 & & + & 1 & 35,54 & 5.56 & 0,018 & $\mathbf{0 . 0 4 6}$ \\
& 2 & + & + & 2 & 37,33 & 5.77 & 0,056 & 0.143 \\
\hline $466^{*}$ & & & + & 1 & - & 4,86 & 0,028 & $\mathbf{0 . 0 4 6}$ \\
\hline 566 & 1 & + & & 1 & 30,55 & 20,11 & $<0,00001$ & $<0.0001$ \\
& 3 & + & + & 2 & 32,47 & 20,19 & $<0,0001$ & $<\mathbf{0 . 0 0 1}$
\end{tabular}

836 * Given that OTU466 was present in all control females, we excluded the factor "OTU presence in female" from this analysis 


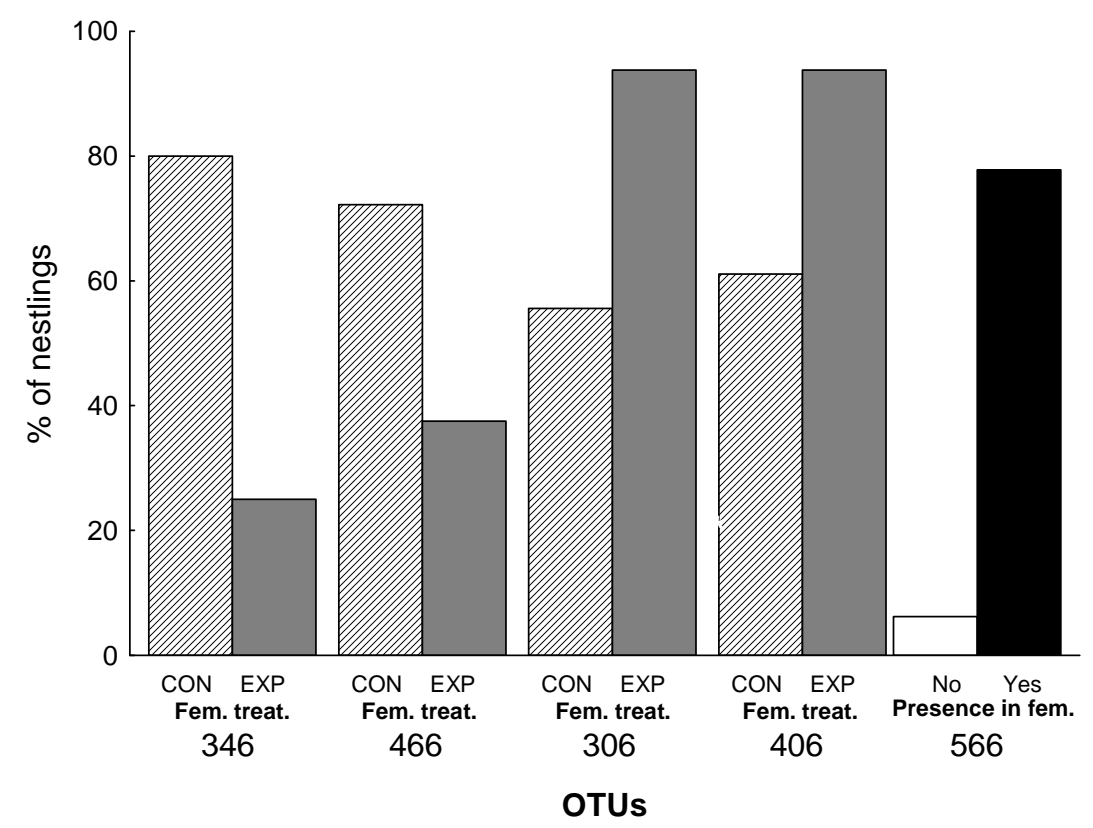

839

840 Figure 3. Influence of the experimental covering of female glands (Fem. treat.) and the presence of an OTU in females at the 841 beginning of incubation (Presence in fem.) on its occurrence in their nestlings at 16 days of age. Graphs show only the effect for the 842 five OTUs for which female manipulation or its presence in females significantly influenced presence in nestling secretions. $\mathrm{CON}=$ 843 Control females. EXP $=$ Experimental females. For OTU346, only the nests with its presence in the female $(9$ females and 18 844 nestlings) are considered (see text), for the remaining OTUs all nests with available information on the microbiome of female 845 secretion are included (17 females and 34 nestlings). The OTU566 was present in nine females and absent in eight females.

848 Occurrence of the OTU566 in the microbiome of nestlings and their mothers were positively associated (Fig. 8493) independently of female experimental treatment $(\mathrm{Wald}=0.077, \mathrm{p}=0.782)$. This result suggests 850 independent acquisition of nestlings and females within the nest environment, not dependent on transmission 851 to nestlings from the mother's gland after female manipulation.

\section{Microbiome enrichment by cross-inoculation}

855 Most cross-inoculated nestlings acquired new OTUs. Indeed, the final samples of cross-inoculated nestlings 856 clearly congregated in the corner of the PCO space correlating with a greater number of OTUs (Fig. 4). The 857 two OTUS whose prevalence in nestlings was reduced by the experiment covering female glands (OTU346 858 and OTU466) were closely associated with this section of the PCO space (Fig. 4) suggesting that they are 859 among the components of the community that explain the detected experimental effects of cross-inoculation. 
860 Moreover, for 10 out of the 14 OTUs of the core microbiome present in females (all except OTU278, 861 OTU310, OTU330, OTU350 and OTU474), there was a significant relationship of the initial differences 862 between receiver and donor and the change in harboring a particular OTU after inoculation (Pearson Chi863 squares, after FDR correction for multiple tests, all $\mathrm{q}<0.05)$. For these ten OTUs, in $92.1 \%$ of the cases in 864 which they were present in a donor and not in the receiver, the inoculated nestling incorporated the OTU to 865 its microbiome.

866 


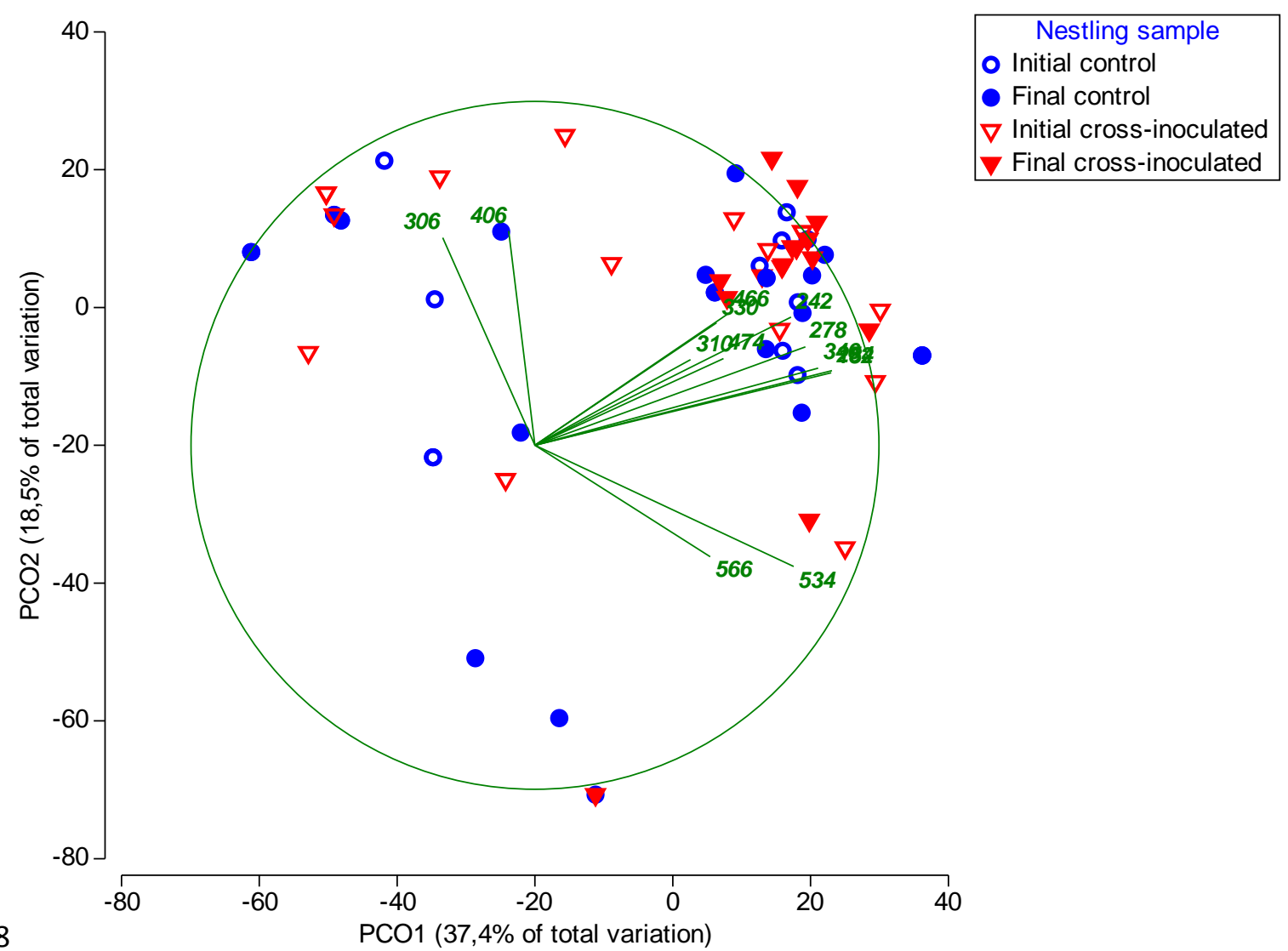

869 Figure 4. PCO plot showing the resemblance between initial and final samples of nestlings for the two groups of the inoculation 870 experiment. The vectors of the correlations of all OTUs with PCO axes are drawn in green.

871

872

873

874 